Archive for

Organic Chemistry
Arkivoc 2017, part iii, 250-268

\title{
Synthesis, structural characterization and cytotoxic activity of heterocyclic compounds containing the furoxan ring
}

\section{Alexander S. Kulikov, ${ }^{a}{ }^{*}$ Alexander A. Larin, ${ }^{a}$ Leonid L. Fershtat, ${ }^{a}$ Lada V. Anikina, ${ }^{b}$ Sergey A. Pukhov, ${ }^{b}$} Sergey G. Klochkov, ${ }^{b}$ Marina I. Struchkova, ${ }^{a}$ Anna A. Romanova, ${ }^{c}$ Ivan V. Ananyev, ${ }^{c}$ and Nina N. Makhova ${ }^{a}$

${ }^{a}$ N. D. Zelinsky Institute of Organic Chemistry, Russian Academy of Sciences, 47 Leninsky prosp., 119991 Moscow, Russian Federation

${ }^{b}$ Institute of Physiologically Active Compounds, Russian Academy of Sciences, 142432 Chernogolovka, Moscow region, Russian Federation

${ }^{c}$ A. N. Nesmeyanov Institute of Organoelement Compounds, Russian Academy of Sciences, 28 Vavilova str., 119991 Moscow, Russian Federation

Email: pvyu@ioc.ac.ru

\section{Dedicated to Prof. Oleg A. Rakitin on the occasion of his 65th birthday}

Received 07-20-2017

Accepted 07-21-2017

Published on line 08-07-2017

\section{Abstract}

A direct approach to the synthesis of previously unknown $1 H-1,2,3$-triazolylfuroxans, involving nucleophilic substitution of the nitro group in nitrofuroxans followed by catalytic [3+2] cycloaddition of intermediate azidofuroxans to 1,3-ketoesters, is reported. The scope of the triazolylfuroxans was additionally diversified through a number of transformations of the functional groups attached to the 1,2,3-triazole ring. The cytotoxic activity of the newly synthesized triazolylfuroxans and of previously reported hetarylfuroxans was studied. The NO-donor capability of selected synthesized hetarylfuroxans was measured by the Griess reaction using a spectrophotometric technique.

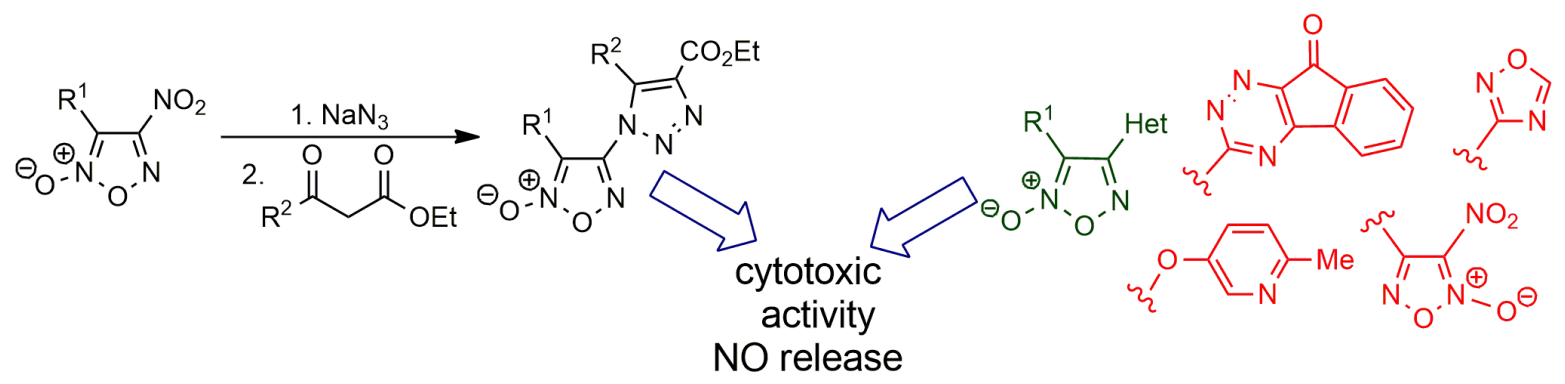

Keywords: Furoxan, 1,2,3-triazole, [3+2] cycloaddition, NO-donor, cytotoxic activity, Griess reaction 


\section{Introduction}

A frequent approach to the design of potential drugs with improved pharmacokinetic profile is based on the molecular hybridization of separate compounds with known pharmacological activity. ${ }^{1-3}$ In particular, in recent years a great attention has been focused on the synthesis of hybrid structures comprising a framework capable of nitric oxide (NO) release. Over thirty years ago it was established that NO is one of the crucial regulator molecules for cellular metabolism, affecting various physiological and pathophysiological processes. ${ }^{4-6}$ Many different types of compounds have been synthesized and tested as NO donors (guanidines, nitramines, oximes, mesoionic systems, heterocyclic $N$-oxides, etc.) ${ }^{7-9}$ including the $1,2,5$-oxadiazole 2 -oxides (furoxans) which are capable of exogenous NO release at the presence of thiol cofactors. ${ }^{10,11}$

Furoxans comprise a valuable class of five-membered heterocycles and can serve as a privileged motif in medicinal and pharmaceutical chemistry owing to their significant biological activities, for example neuroprotective and precognitive, ${ }^{12}$ cytotoxic, ${ }^{13,14}$ antihelmintic, ${ }^{15}$ antibacterial ${ }^{16}$ and fungicidal ${ }^{17}$, connected with the high capacity of furoxans to produce a large flux of NO. It was established that NO exerts a cytotoxic effect at high concentrations, while low levels of NO are potentially protective, particularly in the CNS. ${ }^{18}$ The incorporation of the furoxan ring as a potential NO donor with drug candidates of known pharmacological activity, especially anticancer, has been recently used by many research groups. ${ }^{19-26}$

In present work we have aimed to synthesize the heterocyclic structures comprising furoxan ring coupled with various functionally substituted heterocyclic fragments and to carry out an evaluation of their cytotoxic activity and NO-donor capability.

A series of novel heterocyclic structures containing poly-nitrogen or nitrogen-oxygen heterocycles attached to a furoxan ring either directly or by means of heteroatom bridges: 4-hetaryloxyfuroxans ${ }^{27}$ 1, $(1,2,4-$ oxadiazol-3-yl)furoxans ${ }^{28}$ 2, (1,2,4-triazin-3-yl)furoxans ${ }^{29} \mathbf{3}$ and nitrobifuroxanyl ensembles ${ }^{30} \mathbf{4}$, was available. The compounds 4 have recently been synthesized by our research team. Their antitumor potential had not so far been investigated. In addition, in this work we have developed a general method for the synthesis of the previously unknown (1,2,3-triazol-1-yl)furoxan derivatives 5 (Scheme 1). Various derivatives of these types of heterocycle have previously revealed cytotoxic activity: $1,2,4$-oxadiazole, ${ }^{31} 1,2,4$-triazine, ${ }^{32,33} 1,2,3$-triazole, ${ }^{34}$ and furoxan itself. ${ }^{13,14}$ Heterocyclic motifs, namely quinolines ${ }^{35}$ and pyridines, ${ }^{36}$ connected to the furoxan ring through O-bridges, are also found in compounds possessing cytotoxic activity.

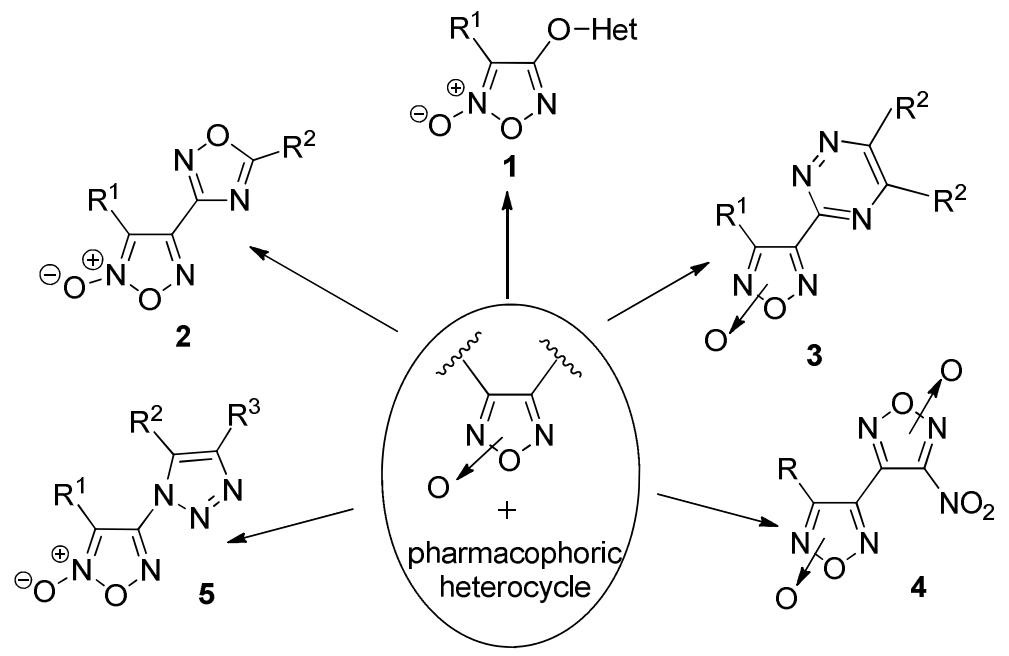

Scheme 1. The investigated heterocyclic structures 1-5, containing a furoxan ring along with different polynitrogen and nitrogen-oxygen heterocycles. 


\section{Results and Discussion}

\section{Synthesis}

The investigations began with the synthesis of the structures 1-5. The compounds 1-4 were prepared according to described procedures. ${ }^{27-30}$ Synthesis of compounds $\mathbf{1 a - d}$ was performed by nucleophilic substitution of the nitro group in readily available ${ }^{37} 4$-nitrofuroxans $\mathbf{6} \mathbf{a}, \mathbf{b}$ by the action of hydroxy-heterocycles (Scheme 2). ${ }^{27}$ The (1,2,4-oxadiazol-3-yl)furoxans $\mathbf{2 a , b}$ were synthesized by means of the solvent-free reaction of the furoxanylamidoximes $\mathbf{7 a , b}$ with trimethyl orthoformate with $\mathrm{Sc}(\mathrm{OTf})_{3}$ catalysis. ${ }^{28}$ Compounds $\mathbf{2 c - f}$ were obtained by means of the tandem heterocyclization of the furoxanylamidoximes $\mathbf{7 a , b}$ with different aromatic carboxylic acid chlorides under mild conditions. ${ }^{28}$ The required amidoximes $7 \mathbf{a}, \mathbf{b}$ were in turn prepared by reaction of the accessible furoxanylcarbonitriles $\mathbf{8 a , b}$ with hydroxylamine (Scheme 3 ).
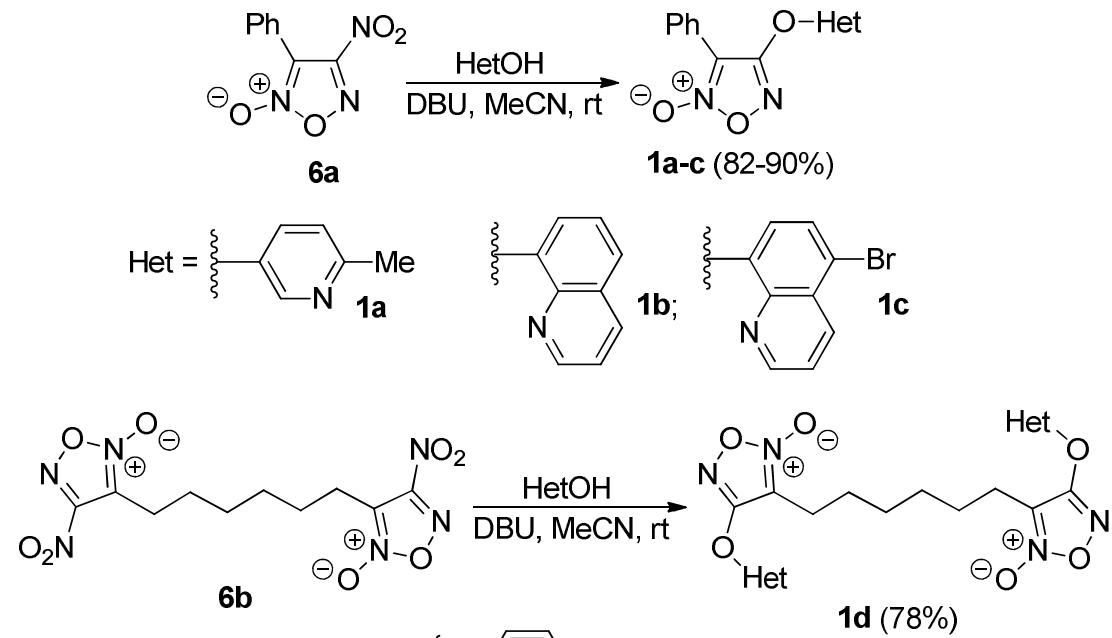

Scheme 2. Synthesis of hetaryloxyfuroxans 1a-d.

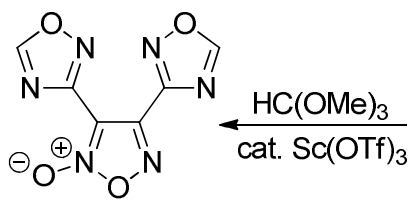

$2 \mathbf{b}(82 \%)$

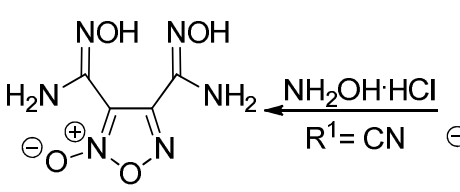

$7 \mathbf{b}(87 \%)$

$\downarrow \begin{gathered}\mathrm{R}^{2} \mathrm{COCl}, \mathrm{Cs}_{2} \mathrm{CO}_{3} \\ \mathrm{MeCN}, \mathrm{rt}\end{gathered}$<smiles>[R]c1nc(-c2[R10]([O-])onc2/C(C)=N/O)no1</smiles><smiles>Cc1ccc(C(C)(C)C)cn1</smiles>

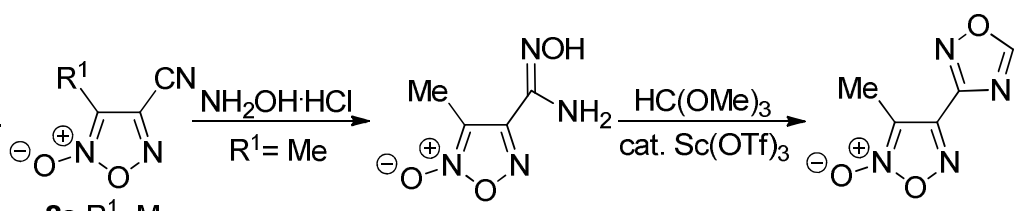

8a $\mathrm{R}^{1}=\mathrm{Me}$, $8 b R^{1}=C N$

$7 a(94 \%)$ $\downarrow \begin{gathered}\mathrm{R}^{2} \mathrm{COCl}, \mathrm{Cs}_{2} \mathrm{CO}_{3} \\ \mathrm{MeCN}, \mathrm{rt}\end{gathered}$<smiles></smiles>

$\mathrm{R}^{2}=2-\mathrm{NO}_{2} \mathrm{C}_{6} \mathrm{H}_{4}(\mathbf{c}) \cdot 3-\mathrm{NO}_{2} \mathrm{C}_{6} \mathrm{H}_{4}(\mathbf{d}), 4-\mathrm{NO}_{2} \mathrm{C}_{6} \mathrm{H}_{4}(\mathbf{e}, \mathbf{f})$

Scheme 3. Synthesis of (1,2,4-oxadiazol-3-yl)furoxans 2a-f. 
For the preparation of (1,2,4-triazin-3-yl)furoxans 3a-g a cyclocondensation of $\alpha$-dicarbonyl compounds 9 with furoxanylamidrazones $\mathbf{1 0}$ was utilized. ${ }^{29}$ An effective synthesis of the latter was recently developed by reaction of cyanofuroxans $\mathbf{8}$ with hydrazine-hydrate (Scheme 4). ${ }^{38}$ The 3-nitrobifuroxanyl structures 4 a-c were synthesized by an interaction of furoxanylhydroxamic acid chlorides $\mathbf{1 1}$ with dinitromethane sodium salt with subsequent nitrosation (Scheme 5). ${ }^{30}$ Compound $\mathbf{4 c}$ was thermally isomerized to the 4-nitro isomer $\mathbf{4 d}$, and 4,4'-dinitro-3,3'-bifuroxan 4 e was prepared according to Klapötke's procedure. ${ }^{39}$

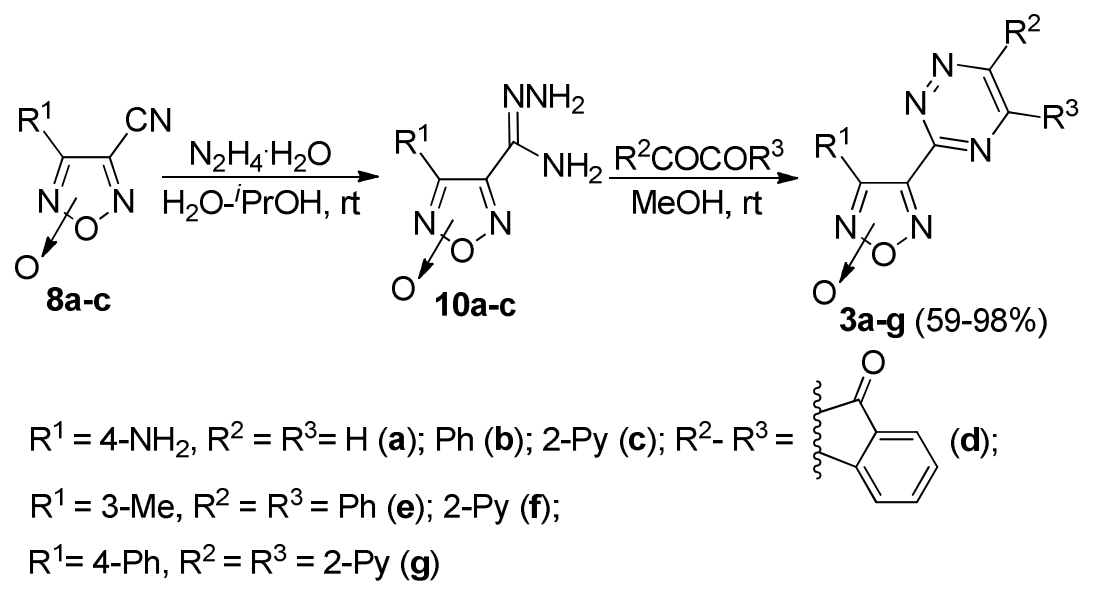

Scheme 4. Synthesis of (1,2,4-triazin-3-yl)furoxans 3a-g $R^{2} \operatorname{COCOR}^{3}$.
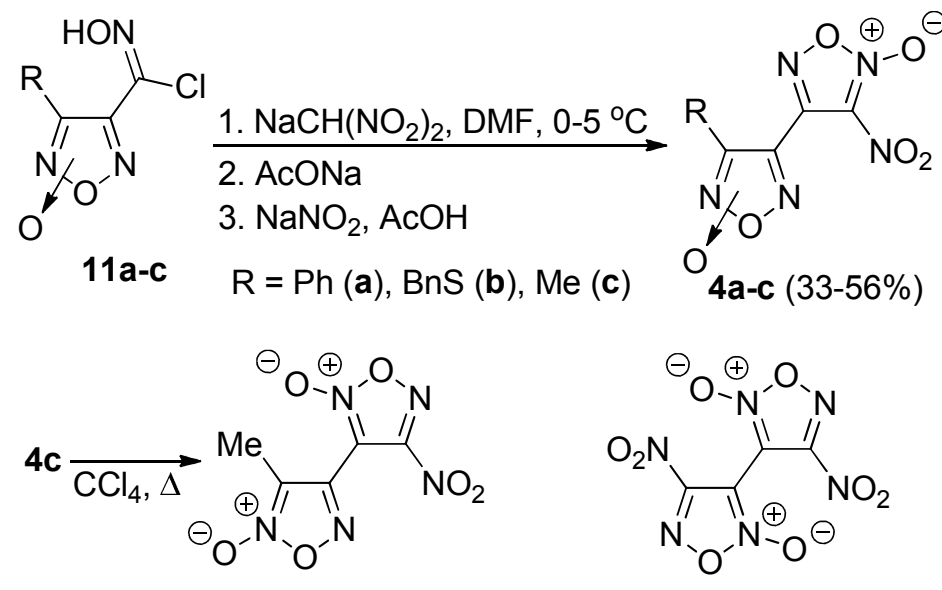

4d $(72 \%)$

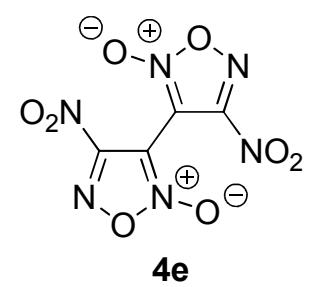

Scheme 5. Synthesis of bifuroxans 4.

To prepare the (1,2,3-triazol-1-yl)furoxan derivatives 5 we applied the approach based on the transformations of chloromethyl and ethoxycarbonyl groups in 1,2,3-triazoles 12a-c by the action of different nucleophiles. The initial compounds $12 \mathrm{a}-\mathrm{c}$ were synthesized by [3+2] cycloaddition of 3-aryl-4-azidofuroxans $\mathbf{1 3 a , b}$ with benzoylacetic ethyl ester $\mathbf{1 4 a}$ and chloroacetoacetic ester $\mathbf{1 4 b}$ under TEA catalysis (Scheme 6 ). This approach was previously used for the synthesis of similar (1,2,3-triazol-1-yl)furazan derivatives which were shown to possess cytotoxic activity. ${ }^{40}$ The initial 3-aryl-4-azidofuroxans $\mathbf{1 3 a , b}$ were prepared by nucleophilic substitution of nitro group in 3-aryl-4-nitrofuroxans 6a,c under the action of $\mathrm{NaN}_{3}$ according to described method. ${ }^{34}$ The nucleophilic substitution of chloride fragment in compounds $\mathbf{1 2} \mathbf{b}, \mathbf{c}$ under the action of 
cycloaliphatic amines and heterocyclic thiols or hydroxyheterocycles resulted in aminoderivatives 15a-c and the (1,2,3-triazol-1-yl)furoxans 5a-f in high yields (Scheme 6).

Cycloalkylamino derivatives 15a-c were consecutively transformed into hydrazides 16a-c, azidocarbonyl derivatives $\mathbf{1 7 a , b}$ and the target (1,2,3-triazol-1-yl)furoxans $\mathbf{5 g}$ and $\mathbf{5 m}$. The azidocarbonylfuroxan $\mathbf{1 7 c}$ was hydrolyzed in situ in the course of its preparation into the acid $5 \mathrm{~m}$. The hydrazide 16 a serves as the initial compound for the synthesis of hydrazone $\mathbf{5 h}$, and the amides $\mathbf{5 i , j , l}$ were prepared from the corresponding azidocarbonyl derivatives $\mathbf{1 7 a , b}$. The hydrolysis of the ethoxycarbonyl group was found to proceed only for compound 12a, with formation of carboxytriazolyl derivative 18. Decarboxylation of this compound as well as the acid $\mathbf{5 g}$ resulted in the (1,2,3-triazol-1-yl)furoxans $\mathbf{5 k}$ and $\mathbf{5 n}$ (Scheme 7).

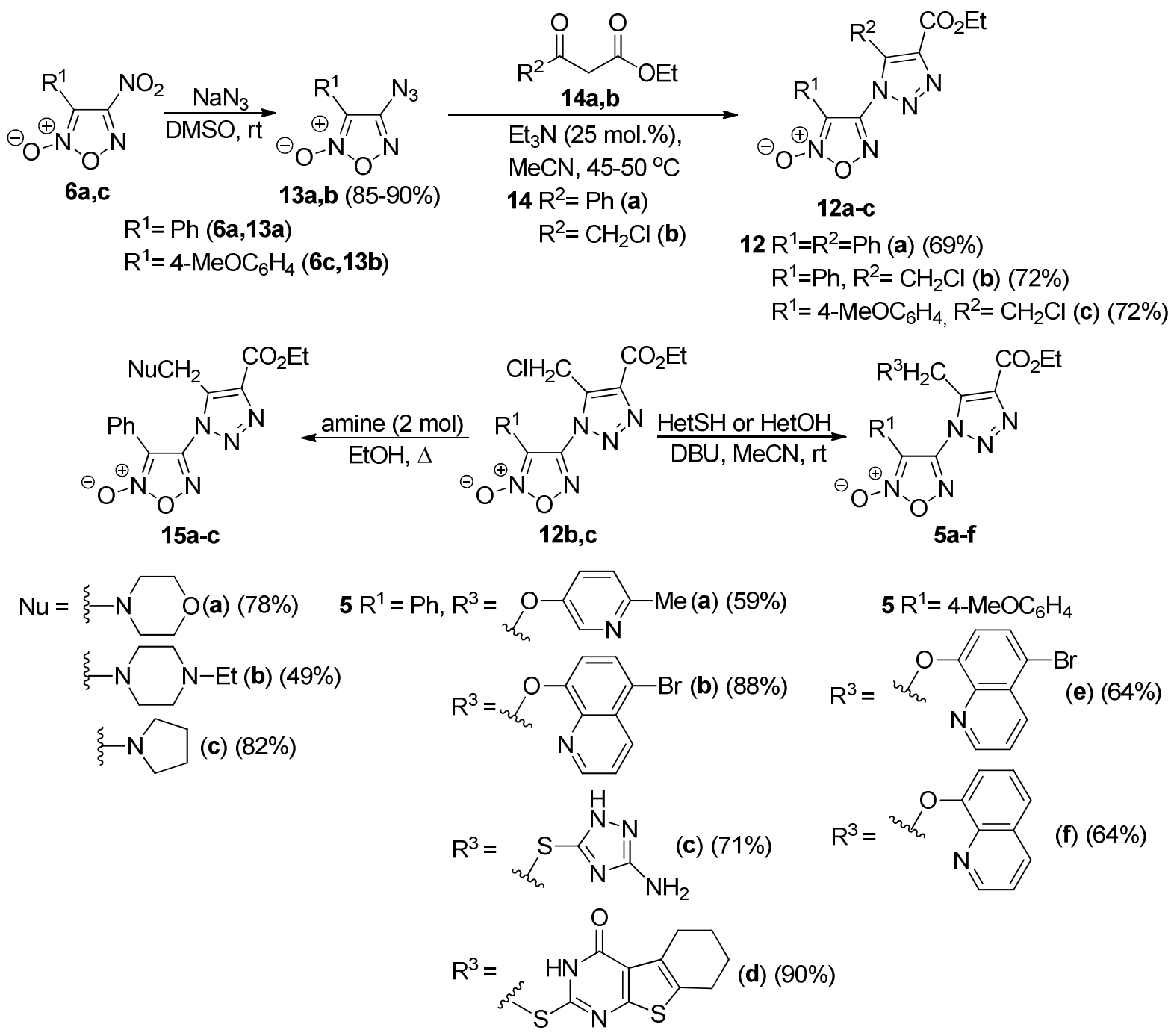

Scheme 6. [3+2] Cycloaddition of 1,3-ketoesters to azidofuroxans and transformations of a chloromethyl substituent in (1,2,3-triazolyl)furoxans $\mathbf{1 2 b , c}$. 


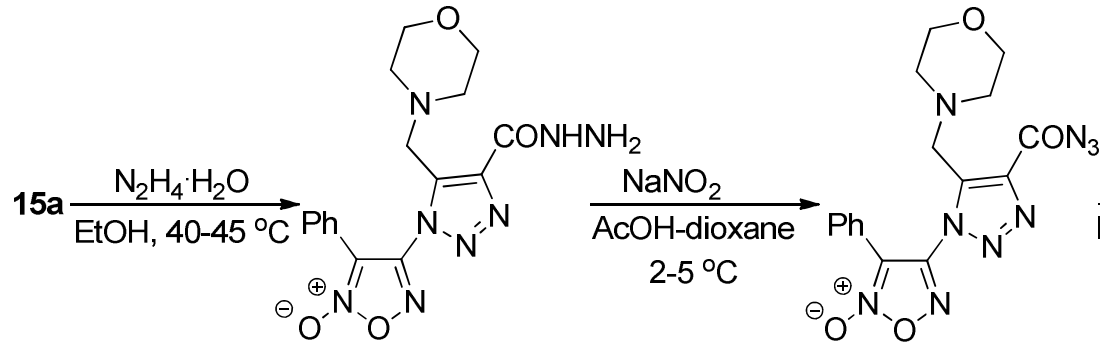<smiles>[134Os]</smiles><smiles>CCCC</smiles><smiles>[BiH3]</smiles><smiles>CCN1C(=O)C(=O)c2cc(C)ccc21</smiles>
$\mathrm{EtOH}-\mathrm{AcOH}, \Delta$<smiles>CCN1C(=O)/C(=N\NC(=O)c2nnn(-c3no[n+]([O-])c3-c3ccccc3)c2CN2CCOCC2)c2cc(Br)ccc21</smiles>

17 a $(92 \%)$ amine (2 mol) dioxane, rt<smiles>NC(=O)c1nnn(-c2no[n+]([O-])c2-c2ccccc2)c1CN1CCOCC1</smiles>

$5 \mathbf{i}, \mathbf{j}$<smiles>CCN1CCN(CC=[Nb])CC1</smiles><smiles>CN1CCCC1</smiles>

$\underset{\mathrm{H}_{2} \mathrm{O}-\text { dioxane, } \mathrm{rt}}{\longrightarrow}$<smiles>[O-][n+]1onc(N2CCN=N2)c1-c1ccccc1</smiles>

$5 \mathrm{~g}(92 \%)$

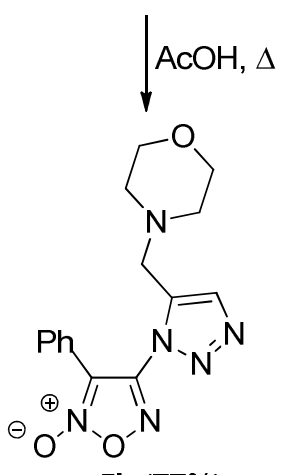

5k $(77 \%)$

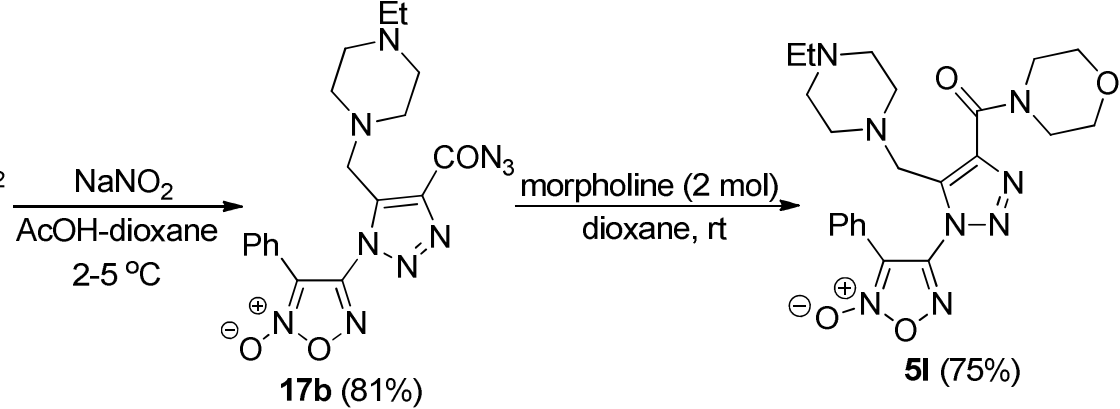

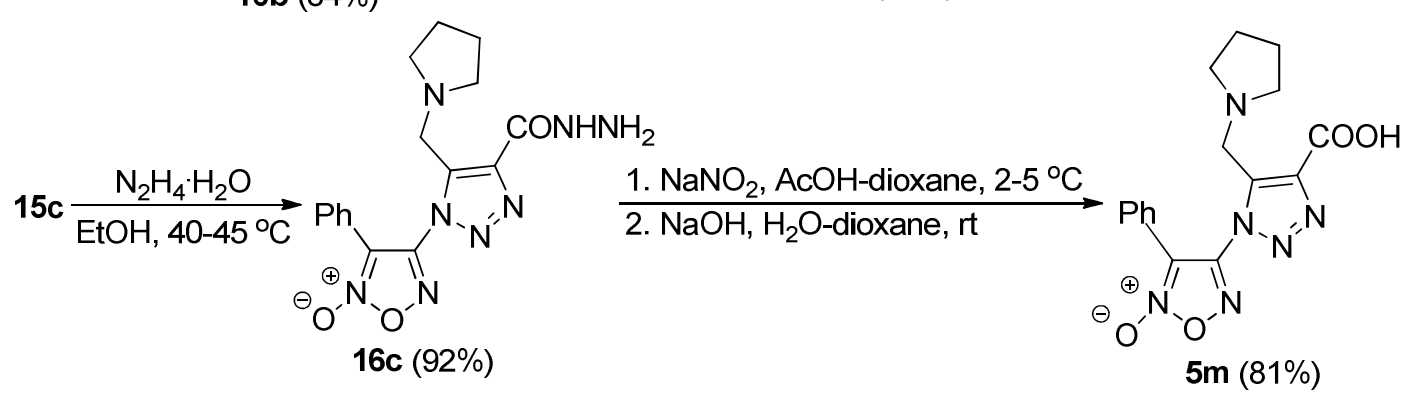

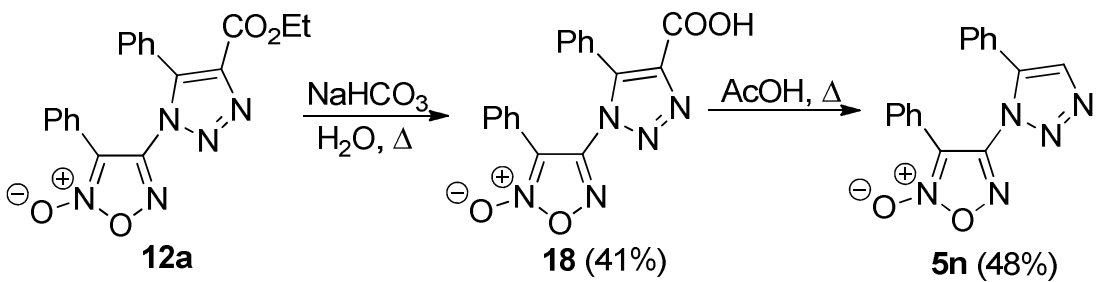

Scheme 7. Transformations of ester group in newly synthesized (1,2,3-triazolyl)furoxans. 
All synthesized intermediate products 12-18 and final (1,2,3-triazol-1-yl)furoxans 5a-n were characterized by spectral (IR, ${ }^{1} \mathrm{H},{ }^{13} \mathrm{C} N M R$ and mass-spectra) and analytical methods. Finally, the structures of the (1,2,3triazol-1-yl)furoxans $\mathbf{5}$ was confirmed by the single-crystal X-ray diffraction study of compound $\mathbf{5 k}$ (Figure 1).

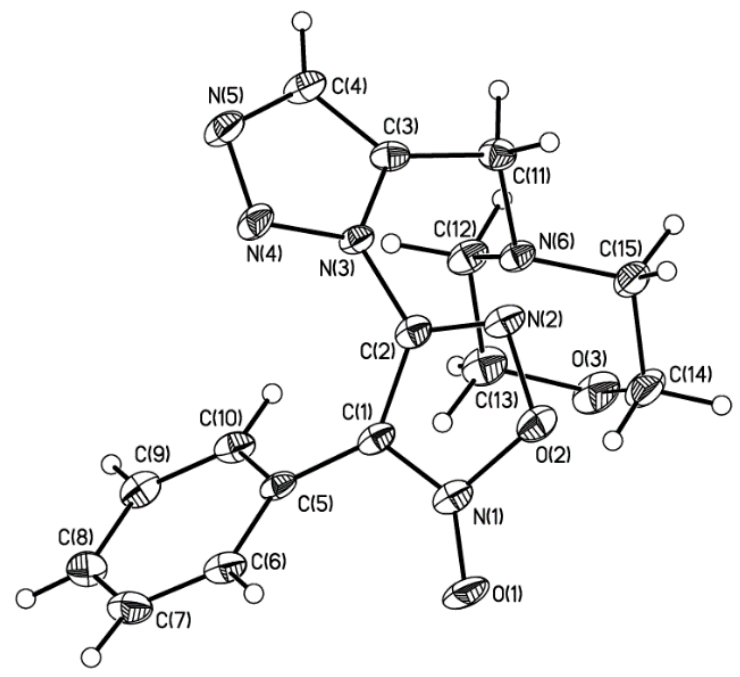

Figure 1. The general view of the $\mathbf{5 k}$ molecule. Atoms are represented by probability ellipsoids of atomic vibrations $(p=0.5)$.

According to X-ray diffraction data the morpholine ring in $\mathbf{5 k}$ adopts a stable chain conformation with practically equal displacements of the $\mathrm{N}(6)$ and $\mathrm{O}(3)$ atoms from the mean-square plane of the cycle $(-0.686(2)$ and $0.631(8) \AA$, respectively). All cyclic fragments in 5k are non-coplanar to each other: the torsion angle $C(2) C(1) C(5) C(10)$ is $42.11(2)^{\circ}$, while the $C(1) C(2) N(3) N(4)$ angle is $55.55(2)^{\circ}$. It indicates the close extent of a $\pi$-conjugation between cycles, that is unusual for substituted phenylfuroxans especially accounting for the acceptor character of triazole ring. The spatial arrangement of cycles in crystal structure of $\mathbf{5 k}$ can be explained not only by the presence of bulky morpholine substituent but also by two intermolecular interactions between cycles bounding molecules into dimers. Namely, there are the $\mathrm{C}-\mathrm{H}$... $\pi$ interaction between hydrogen atom of triazole ring and phenyl cycle (with normalized C-H bonds the $\mathrm{C}(7)$...H(4) distance is $2.757 \AA$ ) and the $\mathrm{C}-\mathrm{H}$...N interaction between hydrogen atom of morpholine cycle and nitrogen of triazole ring (the distance $\mathrm{N}(5) \ldots \mathrm{H}(12 \mathrm{~B})$ is $2.651 \AA$ accounting to normalized $\mathrm{C}-\mathrm{H}$ bonds). Among many other intermolecular contacts, the shortened contacts between the oxygen atom of morpholine cycle and furoxan cycle are to be noted (the $\mathrm{C}(1) \ldots \mathrm{O}(3), \mathrm{N}(1) \ldots \mathrm{O}(3)$ and $\mathrm{C}(2) \ldots \mathrm{O}(3)$ distances are 2.950, 2.970, и $3.158 \AA$, respectively). These contacts are geometrically similar with intermolecular interactions between furoxan ring and its exo-oxygen atom ${ }^{29,41}$ and can be described as interaction between lone electron pair of the $\mathrm{O}(3)$ atom and $\pi^{*}$-orbital of the furoxan cycle. In $\mathbf{5 k}$ these interactions form continuous chains of molecules which are, in its turn, bounded into layers by the $\mathrm{C}-\mathrm{H}$... $\pi$ interaction between $\mathrm{CH}_{2}$-fragment and triazole ring (with normalized $\mathrm{C}-\mathrm{H}$ bonds the $\mathrm{C}(3) \ldots \mathrm{H}(11 \mathrm{~B})$ distance is $2.598 \AA$ ). The crystal packing of $\mathbf{5 k}$ is additionally stabilized by weak $\mathrm{C}-\mathrm{H}$... O contacts between the exo-oxygen atom of furoxan cycle and one of the methylene fragments of morpholine (the $\mathrm{H}(15 \mathrm{~A})$...O(1) distance is $2.539 \AA$ ) (Figure 2). 


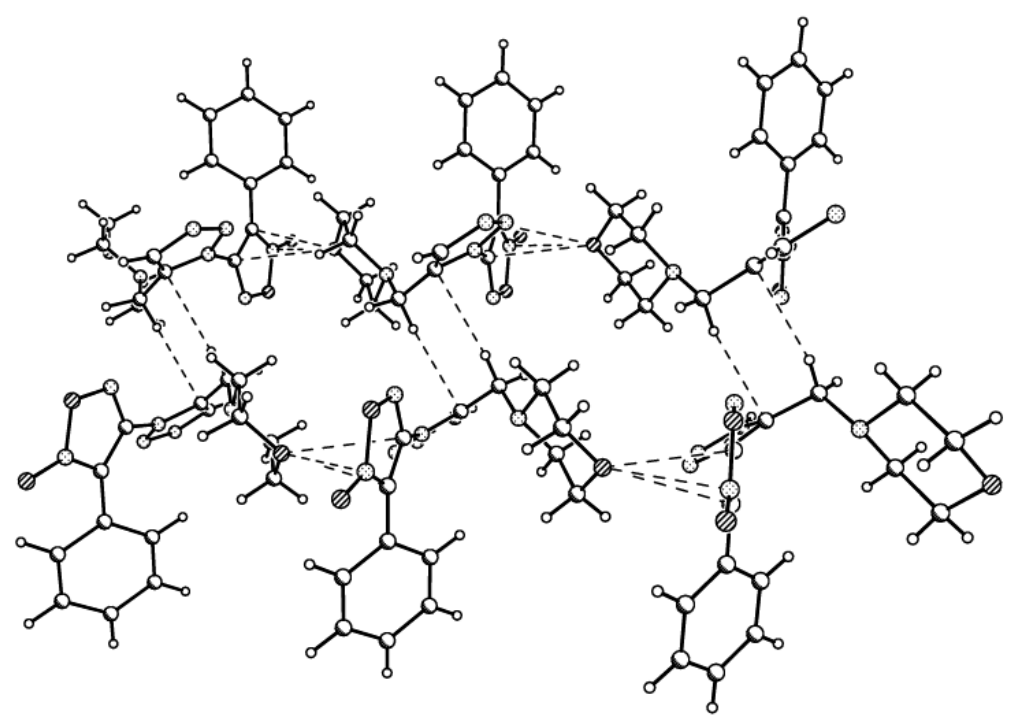

Figure 2. The fragment of a layer in the crystal structure of $\mathbf{5 k}$.

\section{Cytotoxic activity and NO-donating properties}

The cytotoxic activity of compounds 1-5 (36 compounds overall) was tested in vitro by MTT assay against five human cancer cell lines: A549 (lung adenocarcinoma), HCT116 (colon cancer), HeLa (cervical cancer), MCF7 (breast carcinoma), RD (rhabdomyosarcoma). Camptothecin was used as positive control. Cell viability was evaluated after $72 \mathrm{~h}$ of exposure to the compounds at 100-1.56 $\mu \mathrm{M}$ concentrations (Table S1, Supplementary Material). The biological investigations have shown that the most active compounds were 4-(2-methylpyridin5-yloxy)-3-phenylfuroxan (1a), bis(1,2,4-oxadiazolyl)furoxan (2b), 4-amino-3-(indenotriazin-3-yl)furoxan (3d) and nitrobifuroxans 4a-e which exhibited good cytotoxic activity against all studied human cancer cell lines. These compounds could be considered as promising structural scaffolds for further optimization for future biological insights.

It is well-known that furoxans behave as NO donors in presence of thiol cofactors. ${ }^{5,10,11}$ At the same time, the formation of nitrite-anion as a result of NO oxidation may be quantified according to Griess assay and thus may serve as a reliable tool for measuring the amount of $\mathrm{NO}$ release. The amounts of $\mathrm{NO}_{2}^{-}$produced of the selected hetarylfuroxan structures under physiological conditions ( $\mathrm{pH} 7.4 ; 37^{\circ} \mathrm{C}$ ) after $1 \mathrm{~h}$ incubation were measured via the Griess reaction using a spectrophotometric technique. Furoxan $\mathbf{2} \mathbf{b}$ was found to be the most powerful $\mathrm{NO}$ donor (up to $75.6 \% \mathrm{NO}_{2}^{-}$release, Table 1). Nitrobifuroxans $\mathbf{4 a}, \mathbf{c}, \mathbf{d}$ showed also high levels of $\mathrm{NO}_{2}{ }^{-}$ release, however, for compound $\mathbf{4} \mathbf{d}$ this high value is connected with the ability of 4-nitrofuroxans to undergo nucleophilic substitution under the action of thiols. The dependence of NO release for compounds $\mathbf{1 a}, \mathbf{2} \mathbf{b}, \mathbf{3 d}$ from time was estimated. It was found that for the furoxans $\mathbf{1 a}$ and $\mathbf{3 d}$ the produced amount of NO slightly differs in time, while for the compound $\mathbf{2} \mathbf{b}$ this range was $5-12 \%$ (Figure 3 ). 
Table 1. Griess test results for the selected hetarylfuroxan structures

\begin{tabular}{cccc}
\hline Compound & $\mathrm{NO}_{2}^{-}, \%$ & Compound & $\mathrm{NO}_{2}{ }^{-}, \%$ \\
\hline $\mathbf{1 a}$ & 13 & $\mathbf{3 g}$ & 15 \\
$\mathbf{2 b}$ & 75 & $\mathbf{4 a}$ & 57 \\
$\mathbf{3 a}$ & 8 & $\mathbf{4 c}$ & 35 \\
$\mathbf{3 b}$ & 11 & $\mathbf{4 d}$ & 68 \\
$\mathbf{3 c}$ & 25 & $\mathbf{5 b}$ & 9 \\
$\mathbf{3 d}$ & 10 & $\mathbf{5 f}$ & 4 \\
$\mathbf{3 f}$ & $\mathbf{2 1}$ & & \\
\hline
\end{tabular}

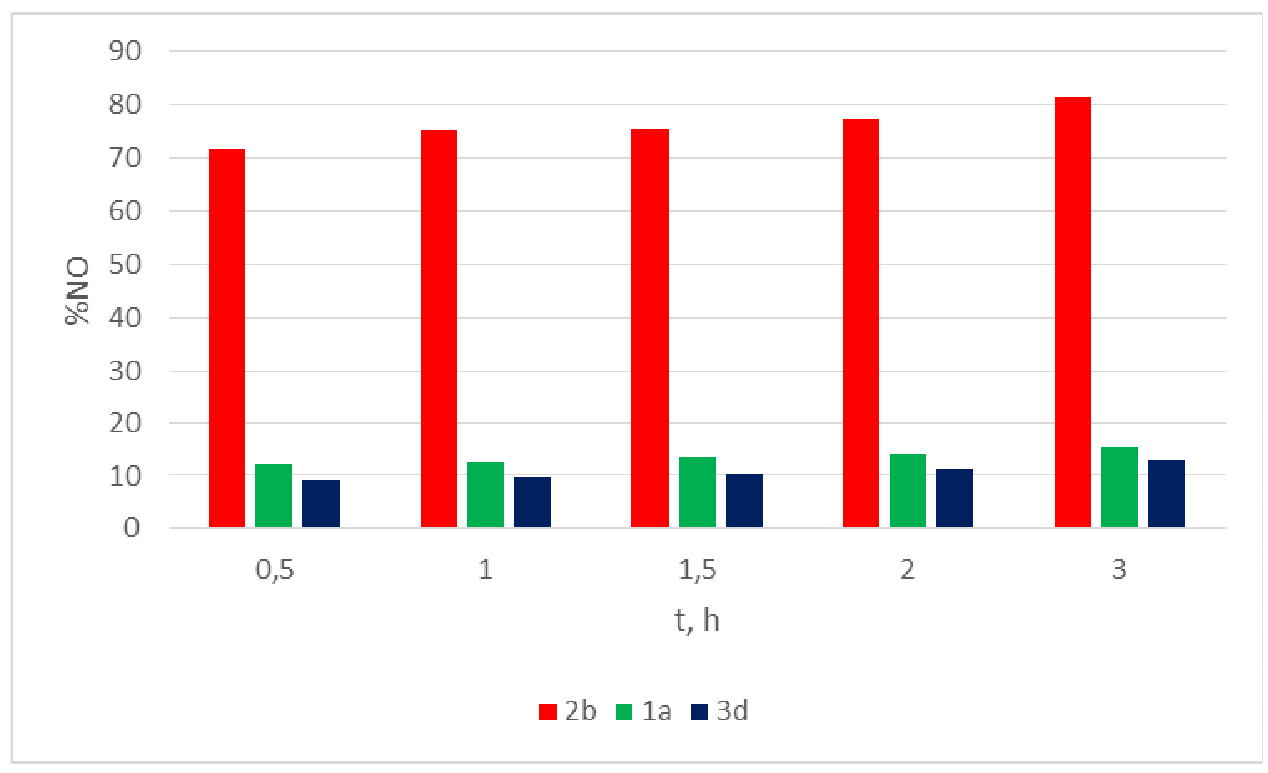

Figure 3. Dependence of $\mathrm{NO}_{2}^{-}$release on time according to Griess test results.

\section{Conclusions}

A novel method for the synthesis of the previously unknown ( $1 \mathrm{H}-1,2,3$-triazolyl)furoxans based on the tandem nucleophilic substitution/organocatalytic [3+2] cycloaddition approach has been developed. The scope of the synthesized heterocyclic assemblies was additionally broadened through the investigations of the reactivity of the functional groups on the triazole ring. A series of newly synthesized ( $1 \mathrm{H}-1,2,3-$ triazolyl)furoxans as well as previously known hetarylfuroxans (36 compounds in total) were evaluated as cytotoxic agents against five human tumor cell lines. In addition, NO-releasing capacity of the selected furoxan-based structures under physiological conditions was measured by detecting nitrites via the Griess reaction using a spectrophotometric technique.

\section{Experimental Section}

General. ${ }^{1} \mathrm{H}$ and ${ }^{13} \mathrm{C}$ NMR spectra were recorded on a Bruker AM-300 (300.13 and $75.47 \mathrm{MHz}$, respectively) spectrometer and referenced to residual solvent peak. ${ }^{14} \mathrm{~N}$ NMR spectra were measured on a Bruker AM-300 
(21.69 MHz) spectrometer using $\mathrm{MeNO}_{2}\left(\delta^{14} \mathrm{~N}=0.0 \mathrm{ppm}\right)$ as an external standard. The chemical shifts are reported in ppm ( $\delta$ ); multiplicities are indicated by $\mathrm{s}$ (singlet), d (doublet), t (triplet), q (quartet), $\mathrm{m}$ (multiplet) and br (broad). Coupling constants, J, are reported in Hertz. The IR spectra were recorded on a Bruker "Alpha" spectrometer in the range $400-4000 \mathrm{~cm}^{-1}$ (resolution $2 \mathrm{~cm}^{-1}$ ) as pellets with $\mathrm{KBr}$ or as a thin layer. The melting points were determined on "Stuart SMP20" melting point apparatus and are uncorrected. Analytical thin-layer chromatography (TLC) was carried out on Merck 25 TLC silica gel $60 \mathrm{~F}_{254}$ aluminum sheets. The visualization of the TLC plates was accomplished with a UV light. Flash chromatography was performed on silica gel $60 \mathrm{~A}$ (0.060-0.200 mm, Acros Organics). High resolution mass spectra were recorded on a Bruker microTOF spectrometer with electrospray ionization (ESI).

\section{Crystallographic data}

Crystals of 5k $\left(\mathrm{C}_{15} \mathrm{H}_{16} \mathrm{~N}_{6} \mathrm{O}_{3}, M=328.34\right)$ are monoclinic, space group $\mathrm{P} 2{ }_{1} / \mathrm{c}$, at $120 \mathrm{~K}: a=9.9763(11), b=$ 8.1184(9), $c=18.998(2) \AA, \beta=95.236(3)^{\circ}, V=1532.2(3) \AA^{3}, Z=4 \quad d_{c a l c}=1.423 \mathrm{~g} \cdot \mathrm{cm}^{-3}, \mu=1.04 \mathrm{~mm}^{-1}, F(000)=$ 688. Intensities of 19922 reflections were measured with a Bruker APEX II CCD diffractometer $[\lambda(\operatorname{MoK} \alpha)=$ $0.71072 \AA, \omega$-scans, $2 \theta<61^{\circ}$ ] and 4535 independent reflections [Rint $=0.0485$ ] were used in further refinement. The structure was solved by direct method and refined by the full-matrix least-squares technique against $F^{2}$ in the isotropic-anisotropic approximation. The hydrogen atoms were found in difference Fourier synthesis and refined in the isotropic approximation. For $\mathbf{5 k}$, the refinement converged to $W R 2=0.1132$ and GOF $=1.023$ for all independent reflections ( $R 1=0.0449$ was calculated against $F$ for 3338 observed reflections with $1>2 \sigma(I)$ ). All calculations were performed using SHELX 2014. ${ }^{59,60}$ CCDC 1536372 contains the supplementary crystallographic data for 5k. These data can be obtained free of charge from deposit@ccdc.cam.ac.uk, through http://www.ccdc.cam.ac.uk/conts/retrieving.html, or from the CCDC, 12 Union Road, Cambridge, CB21EZ, UK.

Synthesis of azidofuroxan 13b. Sodium azide $(1.63 \mathrm{~g}, 25 \mathrm{mmol})$ was added in one portion to a magnetically stirred solution of 4-nitrofuroxan $6 \mathrm{c}(10 \mathrm{mmol})$ in DMSO $(15 \mathrm{~mL})$ at room temperature. The mixture was stirred for $3 \mathrm{~h}$ until disappearance of the initial compound $6 \mathrm{c}$ ( $\mathrm{TLC}$ monitoring, eluent $\mathrm{CHCl}_{3}-\mathrm{CCl}_{4}=1: 1$ ). Then the reaction mixture was diluted with water $(30 \mathrm{~mL})$, the solid formed was filtered off, washed with water and dried in air. Yellow solid; yield $2.24 \mathrm{~g}(96 \%), \mathrm{mp} 103-105{ }^{\circ} \mathrm{C}, \mathrm{R}_{f} 0.71\left(\mathrm{CHCl}_{3}\right)$. IR ( $\left.\mathrm{KBr}\right): 2924,2856,2170,1650$, $1610,1578,1424,1312,1250,1212,1132,1060,982,860 \mathrm{~cm}^{-1} ;{ }^{1} \mathrm{H} N M R\left(300 \mathrm{MHz}, \mathrm{CDCl}_{3}\right) \delta: 7.99$ (d, $2 \mathrm{H}, \mathrm{Ar},{ }^{3} \mathrm{~J}$

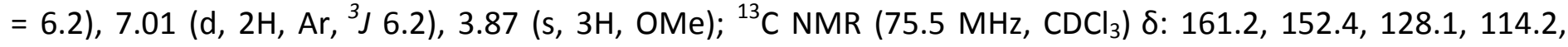
113.3, 108.6, 55.3; ${ }^{14} \mathrm{~N}$ NMR (21.7 MHz, $\left.\mathrm{CDCl}_{3}\right) \delta$ : -145.8 $\left(\mathrm{N}_{3}\right)$. Calcd for $\mathrm{C}_{9} \mathrm{H}_{7} \mathrm{~N}_{5} \mathrm{O}_{3}$ : C, 46.36; $\mathrm{H}, 3.03 ; \mathrm{N}, 30.03$. Found: C, 46.19; H, 2.92; N, 30.17\%.

General procedure for the synthesis of ethyl triazolylfuroxan esters 12a-c. Triethylamine $(0.34 \mathrm{~mL}, 2.5 \mathrm{mmol})$ was added to a solution of the corresponding 3-aryl-4-azidofuroxan $\mathbf{1 3 a , b}(10 \mathrm{mmol})$ and ethyl benzoylacetate 14a (1.91 g, $10 \mathrm{mmol})$ or ethyl chloroacetoacetate 14b (1.65 g, $10 \mathrm{mmol})$ in MeCN (12 mL). The reaction mixture was stirred at $45-50{ }^{\circ} \mathrm{C}$ for $10-16 \mathrm{~h}$ until a disappearance of initial azidofuroxan (TLC monitoring, eluent $\mathrm{CHCl}_{3}$ ). Then $\mathrm{MeCN}$ was evaporated under reduced pressure, $\mathrm{Et}_{2} \mathrm{O}(10 \mathrm{~mL})$ was added and the residue was pounded at cooling. The resulting solid was filtered, washed with a small amount of cold $\mathrm{Et}_{2} \mathrm{O}$ and $\mathrm{dried}$ in air.

Ethyl 5-phenyl-1-(5-oxido-4-phenyl-1,2,5-oxadiazol-5-ium-3-yl)-1H-1,2,3-triazole-4-carboxylate (12a). Light cream solid; yield $2.46 \mathrm{~g}(66 \%), \mathrm{mp} 73-74{ }^{\circ} \mathrm{C}, \mathrm{R}_{f} 0.54\left(\mathrm{CHCl}_{3}\right) . \mathrm{IR}(\mathrm{KBr}): 3384,3324,1736,1609,1535,1503$, 1475, 1445, 1257, 1195, 964, 768, $689 \mathrm{~cm}^{-1} ;{ }^{1} \mathrm{H}$ NMR (300 MHz, CDCl 3 ) $\delta: 7.79(\mathrm{~d}, 1 \mathrm{H}, \mathrm{Ph}), 7.60-7.23(\mathrm{~m}, 6 \mathrm{H}$, $\mathrm{Ph}), 7.18-7.05(\mathrm{~m}, 3 \mathrm{H}, \mathrm{Ph}), 4.35\left(\mathrm{q}, 2 \mathrm{H}, \mathrm{CH}_{2},{ }^{3} \mathrm{~J} 7.1\right), 1.28\left(\mathrm{t}, 3 \mathrm{H}, \mathrm{CH}_{3},{ }^{3} \mathrm{~J} 7.1\right) ;{ }^{13} \mathrm{C} \mathrm{NMR}\left(75.5 \mathrm{MHz}^{\mathrm{CDCl}}\right)_{3} \delta$ : 
$159.8,148.2$, 143.3, 136.8, 131.2, 130.4, 129.5, 129.3, 128.3, 126.9, 126.5, 119.9, 111.1; 61.8, 14.1; HRMS (ESI) $\mathrm{m} / \mathrm{z}$ for $\mathrm{C}_{19} \mathrm{H}_{16} \mathrm{~N}_{5} \mathrm{O}_{4}(\mathrm{M}+\mathrm{H})^{+}$: calcd 378.1197 , found 378.1190 .

Ethyl 5-chloromethyl-1-(5-oxido-3-phenyl-1,2,5-oxadiazol-5-ium-4-yl)-1H-1,2,3-triazole-4-carboxylate (12b). Light cream solid, yield $2.52 \mathrm{~g}(72 \%), \mathrm{mp} 124-125{ }^{\circ} \mathrm{C}, \mathrm{R}_{f} 0,62\left(\mathrm{CHCl}_{3}\right)$. IR (KBr): 3436, 1732, 1614, 1546, 1510, 1482, 1449, 1280, 1216, 1186, 1057, 970, 772, 729, $691 \mathrm{~cm}^{-1} ;{ }^{1} \mathrm{H}$ NMR $\left(300 \mathrm{MHz}, \mathrm{CDCl}_{3}\right) \delta: 7.48$ (br. s, 5H, Ph), $5.18\left(\mathrm{~s}, 2 \mathrm{H}, \mathrm{CH}_{2} \mathrm{Cl}\right), 4.52\left(\mathrm{q}, 2 \mathrm{H}, \mathrm{CH}_{2} \mathrm{CH}_{3},{ }^{3} \mathrm{~J} 7.1 \mathrm{~Hz}\right), 1.48\left(\mathrm{t}, 3 \mathrm{H}, \mathrm{CH}_{3},{ }^{3} \mathrm{~J} 7.1 \mathrm{~Hz}\right) ;{ }^{13} \mathrm{C} \mathrm{NMR}\left(75.5 \mathrm{MHz}, \mathrm{CDCl}_{3}\right) \delta$ : $159.9,147.9,140.1,137.2,131.5,129.4,127.4,120.0,110.8,62.3,30.5,14.2$. HRMS (ESI) $\mathrm{m} / \mathrm{z}$ for $\mathrm{C}_{14} \mathrm{H}_{13}{ }^{35} \mathrm{CIN}_{5} \mathrm{O}_{4}(\mathrm{M}+\mathrm{H})^{+}$: calcd for 350.0651 , found 350.0647 .

Ethyl 5-chloromethyl-1-[3-(4-methoxyphenyl)-5-oxido-1,2,5-oxadiazol-5-ium-4-yl]-1H-1,2,3-triazole-4-carboxylate (12c). Light orange solid, yield $2.73 \mathrm{~g}(72 \%), \mathrm{mp} 219-220{ }^{\circ} \mathrm{C}, \mathrm{R}_{f}=0.66\left(\mathrm{CHCl}_{3}\right) . \mathrm{IR}(\mathrm{KBr}): 3033,2981$, $2848,1735,1607,1520,1469,1429,1378,1299,1212,1180,1155,1014,962,838,741 \mathrm{~cm}^{-1} ;{ }^{1} \mathrm{H}$ NMR (300 MHz, DMSO- $\left.d_{6}\right) \delta: 7.32$ (d, 2H, Ar, ${ }^{3}$ J 8.2), 7.06 (d, 2H, Ar, $\left.{ }^{3} \mathrm{~J} 8.2\right), 5.22\left(\mathrm{~s}, 2 \mathrm{H}, \mathrm{CH}_{2} \mathrm{Cl}\right), 4.42\left(\mathrm{q}, 2 \mathrm{H}, \mathrm{CH}_{2} \mathrm{CH}_{3},{ }^{3} \mathrm{~J}\right.$ 6.5), $3.79\left(\mathrm{~s}, 3 \mathrm{H}, \mathrm{OCH}_{3}\right), 1.37\left(\mathrm{t}, 3 \mathrm{H}, \mathrm{CH}_{2} \mathrm{CH}_{3},{ }^{3} \mathrm{~J} 6.5\right) ;{ }^{13} \mathrm{C} \mathrm{NMR}\left(75.5 \mathrm{MHz}, \mathrm{DMSO}-d_{6}\right) \delta: 161.8,159.7,148.6$, 141.3, 137.6, 129.8, 129.6, 115.2, 112.2, 62.1, 55.9, 31.4, 14.5. HRMS (ESI) $\mathrm{m} / \mathrm{z}$ for $\mathrm{C}_{15} \mathrm{H}_{15}{ }^{35} \mathrm{CIN}_{5} \mathrm{O}_{5}(\mathrm{M}+\mathrm{H})^{+}$: calcd for 380.0757 , found 380.0752 .

General procedure for the synthesis of ethyl aminomethyltriazolyl furoxan esters 15a-c. To a solution of chloromethyl derivative $12 \mathbf{b}(10 \mathrm{mmol})$ in $\mathrm{EtOH}(70 \mathrm{~mL})$ corresponding cycloalkylamine $(20 \mathrm{mmol})$ was added. The reaction mixture was refluxed for $1.5-2.5 \mathrm{~h}$ (TLC monitoring), then cooled to $3-5{ }^{\circ} \mathrm{C}$. The precipitated solid was filtered off, washed with a small amount of cold EtOH, and dried in air.

Ethyl 5-morpholinomethyl-1-(5-oxido-3-phenyl-1,2,5-oxadiazol-5-ium-4-yl)-1H-1,2,3-triazole-4-carboxylate (15a). White solid, yield $3.12 \mathrm{~g}(78 \%), \mathrm{mp} 126-127{ }^{\circ} \mathrm{C}, \mathrm{R}_{f}$ 0,19 $\left(\mathrm{CHCl}_{3}\right)$. IR ( $\left.\mathrm{KBr}\right): 3423,2930,2829,1723,1611$, 1540, 1450, 1226, 1118, 1064, 1013, 955, 868, 775, $701 \mathrm{~cm}^{-1} ;{ }^{1} \mathrm{H} \mathrm{NMR}\left(300 \mathrm{MHz}_{\mathrm{CDCl}}\right) \delta: 7.46$ (s, 5H, Ph), 4.49 (q, $2 \mathrm{H}, \mathrm{OCH}_{2} \mathrm{CH}_{3},{ }^{3}$ J 7.1), 3.94 (s, $2 \mathrm{H}, \mathrm{CH}_{2}$-Triaz.), 3.20 (s, $4 \mathrm{H}, \mathrm{CH}_{2} \mathrm{OCH}_{2}$ ), 2.23 (br. s, $4 \mathrm{H}, \mathrm{CH}_{2} \mathrm{NCH}_{2}$ ), 1.47 (t, $\left.3 \mathrm{H}, \mathrm{OCH}_{2} \mathrm{CH}_{3},{ }^{3} \mathrm{~J} 7.1\right) ;{ }^{13} \mathrm{C} \mathrm{NMR}\left(75.5 \mathrm{MHz}, \mathrm{CDCl}_{3}\right) \delta: 160.5,149.1,142.3,137.9,131.6,129.5,126.5,120.8$, 111.3, 66.3, 62.0, 53.3, 50.0, 14.3. HRMS (ESI) $m / z$ for $\mathrm{C}_{18} \mathrm{H}_{21} \mathrm{~N}_{6} \mathrm{O}_{5}(\mathrm{M}+\mathrm{H})^{+}$: calcd for 401.1568, found 401.1560.

Ethyl 5-[(4-ethylpiperazin-1-yl)methyl]-1-(5-oxido-3-phenyl-1,2,5-oxadiazol-5-ium-4-yl)-1H-1,2,3-triazole-4carboxylate (15b). White solid, yield $2.10 \mathrm{~g}(49 \%), \mathrm{mp} 92-93{ }^{\circ} \mathrm{C}, \mathrm{R}_{f}$ 0,52 $\left(\mathrm{CHCl}_{3}-\mathrm{MeOH}=6: 1\right) . \mathrm{IR}(\mathrm{KBr}): 3412$, 2944, 2824, 1716, 1616, 1543, 1444, 1307, 1230, 1014, 953, 772, $698 \mathrm{~cm}^{-1} ;{ }^{1} \mathrm{H} \mathrm{NMR}\left(300 \mathrm{MHz}, \mathrm{CDCl}_{3}\right) \delta: 7.44$ (s, 5H, Ph), 3.90 (s, 2H, CH $2-$ Triaz.), 4.47 (q, $\left.2 \mathrm{H}, \mathrm{OCH}_{2} \mathrm{CH}_{3},{ }^{3} \mathrm{~J} 7.1\right), 2.27-1.96\left(\mathrm{~m}, 10 \mathrm{H}, \mathrm{N}\left(\mathrm{CH}_{2} \mathrm{CH}_{2}\right)_{2} \mathrm{~N}+\mathrm{CH}_{3} \mathrm{CH}_{2} \mathrm{~N}\right)$, 1.44 (t, 3H, OCH $\mathrm{CH}_{3},{ }^{3}$ J 7.1), $0.95\left(3 \mathrm{H}, \mathrm{t}, \mathrm{CH}_{3} \mathrm{CH}_{2} \mathrm{~N}^{3} \mathrm{~J} 7.0\right) ;{ }^{13} \mathrm{C} \mathrm{NMR}\left(75.5 \mathrm{MHz}, \mathrm{CDCl}_{3}\right) \delta: 160.6,149.3,142.9$, 137.7, 131.5, 129.5, 126.6, 120.9, 111.5, 61.9, 53.1, 52.1, 49.9, 14.3, 11.8. HRMS (ESI) $m / z$ for $\mathrm{C}_{20} \mathrm{H}_{26} \mathrm{~N}_{7} \mathrm{O}_{4}$ $(\mathrm{M}+\mathrm{H})^{+}$: calcd for 428.2040 , found 428.2037 .

Ethyl 1-(5-oxido-3-phenyl-1,2,5-oxadiazol-5-ium-4-yl)-5-(pyrrolidin-1-yl)methyl-1H-1,2,3-triazole-4-carboxylate (15c). White solid, yield $3.15 \mathrm{~g}(82 \%), \mathrm{mp} 107-108{ }^{\circ} \mathrm{C}, \mathrm{R}_{f} 0.36\left(\mathrm{CHCl}_{3}\right) . \mathrm{IR}(\mathrm{KBr}): 2970,2876,2813,1716$, $1615,1548,1447,1378,1304,1225,1187,1078,955,773,698 \mathrm{~cm}^{-1} ;{ }^{1} \mathrm{H} N M R\left(300 \mathrm{MHz}, \mathrm{CDCl}_{3}\right) \delta: 7.42(\mathrm{~s}, 5 \mathrm{H}$, $\mathrm{Ph}$ ), 4.46 (q, $2 \mathrm{H}, \mathrm{CH}_{2} \mathrm{CH}_{3},{ }^{3}$ J 7.1), 4.03 (s, $2 \mathrm{H}, \mathrm{CH}_{2}$-Triaz.), 2.18 (br. s, $\left.4 \mathrm{H}, \mathrm{CH}_{2} \mathrm{CH}_{2} \mathrm{NCH}_{2} \mathrm{CH}_{2}\right), 1.48-1.42\left(\mathrm{~m}, 7 \mathrm{H}, \mathrm{CH}_{3}\right.$ $\left.+\mathrm{CH}_{2} \mathrm{CH}_{2} \mathrm{NCH}_{2} \mathrm{CH}_{2}\right) ;{ }^{13} \mathrm{CNMR}\left(75.5 \mathrm{MHz}, \mathrm{CDCl}_{3}\right) \delta: 160.6,149.2,144.0,136.8,131.3,129.3,126.6,120.9,111.6$, $61.8,53.9,46.9,23.4$ 14.3. HRMS (ESI) $\mathrm{m} / z$ for $\mathrm{C}_{18} \mathrm{H}_{21} \mathrm{~N}_{6} \mathrm{O}_{4}(\mathrm{M}+\mathrm{H})^{+}$: calcd for 385.1619 , found 385.1613.

General procedure for the synthesis of ethyl (hetaryloxy)methyltriazolyl furoxan esters 5a,b,e,f. Diazabicycloundecene (DBU) $(0.80 \mathrm{~g}, 0.52 \mathrm{mmol})$ was added to a solution of corresponding hydroxyhetarene $(0.52 \mathrm{mmol})$ in $\mathrm{MeCN}(3 \mathrm{~mL})$ at room temperature. Then the chloromethyl derivative $12 \mathrm{~b}$ or $12 \mathrm{c}(0.52 \mathrm{mmol})$ was added. The reaction mixture was stirred at room temperature for 24-72 $\mathrm{h}$ until disappearance of the initial compound 12b or 12c (TLC monitoring). Water (15 mL) was added, the solid formed was filtered off, washed with small amount of cold $\mathrm{CHCl}_{3}$ and dried in air. 
Ethyl 5-(6-methylpyridin-3-yloxy)methyl-1-(5-oxido-3-phenyl-1,2,5-oxadiazol-5-ium-4-yl)-1H-1,2,3-triazole-4carboxylate (5a). Light orange solid, yield $0.10 \mathrm{~g}(59 \%), \mathrm{mp} 123-124{ }^{\circ} \mathrm{C}, \mathrm{R}_{f} 0.16\left(\mathrm{CHCl}_{3}\right)$. IR (KBr): 3422, 2924, $2855,1720,1617,1577,1546,1510,1477,1448,1389,1306,1268,1229,1190,1085,1048,1006,820 \mathrm{~cm}^{-1}$; ${ }^{1} \mathrm{H}$ NMR $\left(300 \mathrm{MHz}, \mathrm{DMSO}-d_{6}\right) \delta$ : 7.54-7.47 (m, 4H, Ar), 7.36-7.33 (m, 2H, Ar), 7.21-7.12 (m, 2H, Ar), $5.60(\mathrm{~s}, 2 \mathrm{H}$, $\mathrm{CH}_{2}$ Triaz.), 4.37 (q, $2 \mathrm{H}, \mathrm{OCH}_{2} \mathrm{CH}_{3},{ }^{3}$ J 7.4), $2.36\left(\mathrm{~s}, 3 \mathrm{H}, \mathrm{CH}_{3}\right), 1.27$ (t, 3H, $\left.\mathrm{OCH}_{2} \mathrm{CH}_{3},{ }^{3} \mathrm{~J} 7.4\right) ;{ }^{13} \mathrm{C} \mathrm{NMR}(75.5 \mathrm{MHz}$, DMSO- $\left.d_{6}\right) \delta: 151.5,151.2,148.5,139.7,137.8,136.4,131.5,129.3,127.3,123.4,122.1,120.1,112.0,61.6$, 58.0, 23.0, 13.9. HRMS (ESI) $\mathrm{m} / z$ for $\mathrm{C}_{20} \mathrm{H}_{19} \mathrm{~N}_{6} \mathrm{O}_{5}(\mathrm{M}+\mathrm{H})^{+}$: calcd for 423.1412 , found 423.1409 .

Ethyl 5-(5-bromoquinolin-8-yloxy)methyl-1-(5-oxido-3-phenyl-1,2,5-oxadiazol-5-ium-4-yl)-1H-1,2,3-triazole4-carboxylate (5b). Yellow solid, yield $0.26 \mathrm{~g}(88 \%), \mathrm{mp} 140-141{ }^{\circ} \mathrm{C}, \mathrm{R}_{f} 0.12\left(\mathrm{CHCl}_{3}\right) . \mathrm{IR}(\mathrm{KBr}): 3070,2985,2933$, $1753,1744,1620,1611,1540,1497,1447,1379,1303,1276,1212,1185,1126,1080,817,790 \mathrm{~cm}^{-1} ;{ }^{1} \mathrm{H} \mathrm{NMR}$ (300 MHz, DMSO- $\left.d_{6}\right)$ 8: 8.74-8.72 (m, $\left.1 \mathrm{H}, \mathrm{Ar}\right), 8.38\left(\mathrm{~d}, 1 \mathrm{H}, \mathrm{Ar}^{3}{ }^{3} \mathrm{~J} 8.5\right), 7.83\left(\mathrm{~d}, 1 \mathrm{H}, \mathrm{Ar},{ }^{3} \mathrm{~J}\right.$ 8.5), 7.71-7.65 (m, $1 \mathrm{H}$, $\mathrm{Ar})$, 7.45-7.33 (m, 3H, Ar), 7.19-7.15 (m, 3H, Ar), 5.84 (s, 2H, $\mathrm{CH}_{2}$ Triaz.), $4.43\left(\mathrm{q}, 2 \mathrm{H}, \mathrm{OCH}_{2} \mathrm{CH}_{3}{ }^{3} \mathrm{~J}\right.$ 7.0), $1.37(\mathrm{t}, 3 \mathrm{H}$, $\mathrm{CH}_{3},{ }^{3}$ J 7.0), ${ }^{13} \mathrm{C}$ NMR $\left(75.5 \mathrm{MHz}\right.$, DMSO- $\left.d_{6}\right) \delta: 159.8,152.4,150.5,149.6,140.8,140.2,135.1,131.4,130.3$, 129.3, 127.7, 127.0, 123.8, 120.5, 112.9, 111.1, 61.9, 60.4, 14.3. HRMS (ESI) $\mathrm{m} / \mathrm{z}$ for $\mathrm{C}_{23} \mathrm{H}_{18}{ }^{79} \mathrm{BrN}_{6} \mathrm{O}_{5}(\mathrm{M}+\mathrm{H})^{+}$: calcd for 537.0517 , found 537.0510 .

Ethyl 5-(5-bromoquinolin-8-yloxy)methyl-1-[3-(4-methoxyphenyl)-5-oxido-1,2,5-oxadiazol-5-ium-4-yl]-1H1,2,3-triazole-4-carboxylate (5e). Light grey solid, yield $0.18 \mathrm{~g}(64 \%), \mathrm{mp} 138-139{ }^{\circ} \mathrm{C}, \mathrm{R}_{f} 0.15\left(\mathrm{CHCl}_{3}\right) . \mathrm{IR}(\mathrm{KBr})$ : 3082 , 2980, 2940, 1743, 1633, 1606, 1540, 1486, 1457, 1439, 1358, 1300, 1256, 1215, 1192, 1176, 1125, 1103, $1077,1019,958,914,814,790 \mathrm{~cm}^{-1} ;{ }^{1} \mathrm{H}$ NMR (300 MHz, DMSO-d 6 ) $\delta: 8.74-8.72(\mathrm{~m}, 1 \mathrm{H}, \mathrm{Het}), 8.36$ (d, $1 \mathrm{H}, \mathrm{Het}$ , ${ }^{3}$ 8.5), 7.80 (d, $\left.1 \mathrm{H}, \mathrm{Het},{ }^{3} \mathrm{~J} 8.5\right), 7.69-7.65$ (m, 1H, Het), 7.14 (d, $\left.1 \mathrm{H}, \mathrm{Het},{ }^{3} \mathrm{~J} 8.5\right), 7.06$ (d, 2H, Ar, $\left.{ }^{3} \mathrm{~J} 7.8\right), 6.87$ (d, $2 \mathrm{H}, \mathrm{Ar},{ }^{3} \mathrm{~J} 7.8$ ), 5.87 (s, 2H, CH Triaz.), 4.44 (q, $\left.2 \mathrm{H}, \mathrm{OCH}_{2} \mathrm{CH}_{3}, 3 \mathrm{~J} 7.1\right), 3.73\left(\mathrm{~s}, 3 \mathrm{H}, \mathrm{OCH}_{3}\right), 1.38\left(\mathrm{t}, 3 \mathrm{H}, \mathrm{OCH}_{2} \mathrm{CH}_{3},{ }^{3} \mathrm{~J}\right.$ 7.1); ${ }^{13} \mathrm{C}$ NMR (75.5 MHz, DMSO- $d_{6}$ ) $\delta: 161.1,159.6,152.1,150.2,149.3,140.6,140.0,136.9,134.7,130.0$, $128.2,127.4,123.5,114.6,112.7,111.9,111.8,110.7,61.7,60.0,55.4,14.0$. HRMS (ESI) $\mathrm{m} / \mathrm{z}$ for $\mathrm{C}_{24} \mathrm{H}_{20}{ }^{79} \mathrm{BrN}_{6} \mathrm{O}_{6}(\mathrm{M}+\mathrm{H})^{+}$: calcd for 567.0623 , found 567.0617 .

Ethyl 1-[3-(4-methoxyphenyl)-5-oxido-1,2,5-oxadiazol-5-ium-4-yl]-5-(quinolin-8-yloxy)methyl-1H-1,2,3triazole-4-carboxylate (5f). Light brown solid, yield $0.16 \mathrm{~g}(64 \%), \mathrm{mp} 159-160{ }^{\circ} \mathrm{C}, \mathrm{R}_{f} 0.18\left(\mathrm{CHCl}_{3}\right) . \mathrm{IR}(\mathrm{KBr}): 3436$, $1740,1604,1571,1520,1482,1375,1318,1275,1261,1217,1183,1115,1073,1031,1018,833,791,757 \mathrm{~cm}$

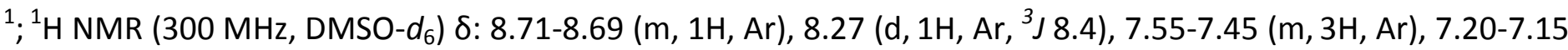

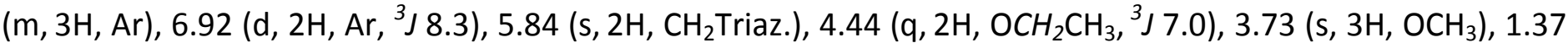
(t, $3 \mathrm{H}, \mathrm{OCH}_{2} \mathrm{CH}_{3},{ }^{3} J$ 7.0); ${ }^{13} \mathrm{C}$ NMR (75.5 MHz, DMSO-d $)$ ) $: 161.5,160.1,152.7,151.3,150.1,150.0,141.3$, $136.3,129.5,129.0,127.0,122.5,121.8,115.2,112.6,112.5,110.4,62.1,60.5,55.9,14.5$. HRMS (ESI) $\mathrm{m} / \mathrm{z}$ for $\mathrm{C}_{24} \mathrm{H}_{21} \mathrm{~N}_{6} \mathrm{O}_{6}(\mathrm{M}+\mathrm{H})^{+}$: calcd for 489.1518 , found 489.1510 .

Synthesis of ethyl 5-(3-amino-1H-1,2,4-triazol-5-ylthio)methyl-1-(5-oxido-3-phenyl-1,2,5-oxadiazol-5-ium-4yl)-1H-1,2,3-triazole-4-carboxylate (5c)

DBU (86 mg, $0.56 \mathrm{mmol}$ ) was added to the solution of the 3-amino-1,2,4-triazole-5-thione (65 mg, $0.56 \mathrm{mmol}$ ) in $\mathrm{MeCN}(3 \mathrm{~mL}$ ). Then compound 12b (196 mg, $0.56 \mathrm{mmol}$ ) was added. The reaction mixture was stirred for 72 $\mathrm{h}$ at room temperature until disappearance of the initial compound $\mathbf{1 2 b}$ (TLC monitoring). Then water (15 $\mathrm{mL}$ ) was added, the resulting mixture was extracted with $\mathrm{CHCl}_{3}(3 \times 20 \mathrm{~mL})$, washed with water and dried over $\mathrm{MgSO}_{4}$. Light yellow solid, yield $0.17 \mathrm{~g}(71 \%)$, mp84-85 ${ }^{\circ} \mathrm{C}, \mathrm{R}_{f} 0.21$ ( $\mathrm{CHCl}_{3}$-EtOAc = 3:1). IR (KBr): 3364, 2982, $2933,1725,1617,1546,1480,1449,1375,1308,1278,1228,1159,1049,968,756,690 \mathrm{~cm}^{-1} .{ }^{1} \mathrm{H}$ NMR (300 $\left.\mathrm{MHz}, \mathrm{DMSO}-d_{6}\right) \delta: 8.31(\mathrm{~s}, 1 \mathrm{H}, \mathrm{NH}) .7 .56-7.45(\mathrm{~m}, 3 \mathrm{H}, \mathrm{Ph}), 7.33\left(\mathrm{~d}, 2 \mathrm{H}, \mathrm{Ph},{ }^{3} \mathrm{~J} 7.6\right), 4.73\left(\mathrm{~s}, 2 \mathrm{H}, \mathrm{CH}_{2} \mathrm{Triaz}\right), 4.35$ (q, $\left.2 \mathrm{H}, \mathrm{OCH}_{2} \mathrm{CH}_{3},{ }^{3} \mathrm{~J} 7.1\right), 1.34\left(\mathrm{t}, 3 \mathrm{H}, \mathrm{OCH}_{2} \mathrm{CH}_{3},{ }^{3} \mathrm{~J} 7.1\right) ;{ }^{13} \mathrm{C} \mathrm{NMR}\left(75.5 \mathrm{MHz}, \mathrm{DMSO}-d_{6}\right) \delta: 159.5,157.7,148.4$, 143.0, 136.9, 131.3, 129.1, 127.6, 127.2, 120.2, 111.4, 61.4, 22.8, 14.0. HRMS (ESI) $\mathrm{m} / z$ for $\mathrm{C}_{16} \mathrm{H}_{16} \mathrm{~N}_{9} \mathrm{O}_{4} \mathrm{~S}$ $(\mathrm{M}+\mathrm{H})^{+}$: calcd for 430.1041 , found 430.1035 . 
Synthesis of ethyl 5-(4-oxo-3,4,5,6,7,8-hexahydrobenzo[4,5]thieno[2,3-d]pyrimidin-2-ylthio)methyl-1-(5oxido-3-phenyl-1,2,5-oxadiazol-5-ium-4-yl)-1H-1,2,3-triazole-4-carboxylate (5d). The ester $12 b$ (200 mg, 0.58 $\mathrm{mmol}$ ) was added to the solution of potassium salt of mercaptohetarene (160 $\mathrm{mg}, 0.58 \mathrm{mmol})$ in DMF (5 mL). The reaction mixture was stirred at room temperature for $24 \mathrm{~h}$. Then water $(25 \mathrm{~mL})$ was added, the solid formed was filtered off, washed with $\mathrm{Et}_{2} \mathrm{O}$ and dried in air. White solid, yield $250 \mathrm{mg}(90 \%), \mathrm{mp}^{229}-230{ }^{\circ} \mathrm{C}, \mathrm{R}_{f}$ $0.08\left(\mathrm{CHCl}_{3}\right)$. IR (KBr): 3059, 2937, 2321, 2840, 1749, 1648, 1618, 1555, 1477, 1448, 1407, 1277, 1197, 1177, 1020, 963, 773, 690, $547 \mathrm{~cm}^{-1} .{ }^{1} \mathrm{H}$ NMR (300 MHz, DMSO-d $)$ ס: 7.48-7.43 (m, 1H, Ph). 7.37-7.33 (m, 2H, Ph), 7.19-7.17 (m, 2H, Ph), 4.97 (s, 2H, $\mathrm{CH}_{2}$ Triaz.), 4.42 (q, $2 \mathrm{H}, \mathrm{OCH}_{2} \mathrm{CH}_{3}{ }^{3}$ J 6.8), 2.72 (br. s, $4 \mathrm{H}, 2 \mathrm{CH}_{2}$ ), 1.73 (br. s, $4 \mathrm{H}$, $2 \mathrm{CH}_{2}$ ), 1.36 (t, $\left.3 \mathrm{H}, \mathrm{OCH}_{2} \mathrm{CH}_{3},{ }^{3} \mathrm{~J} 6.8\right) ;{ }^{13} \mathrm{C} \mathrm{NMR}\left(75.5 \mathrm{MHz}, \mathrm{DMSO}-d_{6}\right) \delta: 160.1,158.2,148.8,142.5,138.0,131.8$, 131.5, 131.1, 129.7, 129.5, 127.5, 127.4, 120.5, 119.8, 111.9, 62.0, 25.6, 24.8, 22.9, 22.2, 22.0, 14.5. HRMS (ESI) $\mathrm{m} / z$ for $\mathrm{C}_{24} \mathrm{H}_{22} \mathrm{~N}_{7} \mathrm{O}_{5} \mathrm{~S}_{2}(\mathrm{M}+\mathrm{H})^{+}$: calcd for 552.1119 , found 552.1113 .

General procedure for the synthesis of furoxancarbohydrazides 16a-c. Hydrazine hydrate $(5 \mathrm{~mL}, 100 \mathrm{mmol})$ was added to a suspension of the corresponding compound 15 (5 mmol) in EtOH (50 mL) at room temperature. The reaction mixture was stirred at $45-50{ }^{\circ} \mathrm{C}$ for $1 \mathrm{~h}$ and at $20{ }^{\circ} \mathrm{C}$ for $10 \mathrm{~h}$ until disappearance of the initial compound 15 (TLC monitoring). Then $\mathrm{H}_{2} \mathrm{O}(75 \mathrm{~mL})$ was added dropwise, the precipitate was filtered off, washed with water, then with small amount of EtOH and dried in air.

5-Morpholinomethyl-1-(5-oxido-3-phenyl-1,2,5-oxadiazol-5-ium-4-yl)-1H-1,2,3-triazole-4-carbohydrazide (16a). White solid, $1.70 \mathrm{~g}(88 \%)$ yield, $\mathrm{mp} 171-172{ }^{\circ} \mathrm{C}, \mathrm{R}_{f} 0.53\left(\mathrm{CHCl}_{3}-\mathrm{MeOH}=6: 1\right)$. IR ( $\left.\mathrm{KBr}\right): 3336,3297,2851$, $1674,1622,1544,1510,1476,1449,1288,1115,956,867,768 \mathrm{~cm}^{-1} ;{ }^{1} \mathrm{H}$ NMR $\left(300 \mathrm{MHz}, \mathrm{CDCl}_{3}\right) \delta: 8.58$ (br. s, 1H, CONH), 7.44 (s, 5H, Ph), 4.13 (br. s, 2H, NH$)_{2}$ ) 3.98 (s, 2H, CH $\mathrm{CH}_{2}$ Triaz.) 3.23 (s, 4H, $\left.\mathrm{CH}_{2} \mathrm{OCH}_{2}\right), 2.25$ (s, 4H, $\left.\mathrm{CH}_{2} \mathrm{NCH}_{2}\right) ;{ }^{13} \mathrm{C}$ NMR $\left(75.5 \mathrm{MHz}, \mathrm{CDCl}_{3}\right) \delta: 160.5,149.2,140.4,138.6,131.6,129.5,126.6,120.8,111.3,66.3$, 53.3, 49.8. HRMS (ESI) $m / z$ for $\mathrm{C}_{16} \mathrm{H}_{19} \mathrm{~N}_{8} \mathrm{O}_{4}(\mathrm{M}+\mathrm{H})^{+}$: calcd for 387.1524 , found 387.1516 .

\section{5-(4-Ethylpiperazin-1-yl)methyl-1-(5-oxido-3-phenyl-1,2,5-oxadiazol-5-ium-4-yl)-1H-1,2,3-triazole-4-carbo-}

hydrazide (16b). White solid, $1.83 \mathrm{~g}(84 \%)$ yield, mp150-151 ${ }^{\circ} \mathrm{C}, \mathrm{R}_{f} 0.45\left(\mathrm{CHCl}_{3}-\mathrm{MeOH}=6: 1\right) . \mathrm{IR}(\mathrm{KBr}): 3411$, $3347,2940,2827,2808,1671,1619,1552,1504,1476,1449,1289,1166,1015,912,775,697 \mathrm{~cm}^{-1} ;{ }^{1} \mathrm{H} \mathrm{NMR}$

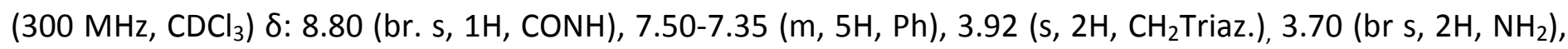
2.27-1.96 (m, $\left.10 \mathrm{H}, \mathrm{N}\left(\mathrm{CH}_{2} \mathrm{CH}_{2}\right)_{2} \mathrm{~N}+\mathrm{CH}_{3} \mathrm{CH}_{2} \mathrm{~N}\right), 0.94\left(\mathrm{t}, 3 \mathrm{H},{ }^{3} \mathrm{~J} 7.1, \mathrm{CH}_{3}\right) ;{ }^{13} \mathrm{C} \mathrm{NMR}\left(75.5 \mathrm{MHz}, \mathrm{CDCl}_{3}\right) \delta: 160.5$, $149.2,140.9,138.3,131.5,129.4,126.5,120.8,111.4,61.9,53.1,52.1,49.6,11.7 . \mathrm{HRMS}$ (ESI) $\mathrm{m} / \mathrm{z}$ for $\mathrm{C}_{18} \mathrm{H}_{24} \mathrm{~N}_{9} \mathrm{O}_{3}(\mathrm{M}+\mathrm{H})^{+}$: calcd for 414.1997, found 414.1991.

1-(5-Oxido-3-phenyl-1,2,5-oxadiazol-5-ium-4-yl)-5-(pyrrolidin-1-yl)methyl-1H-1,2,3-triazole-4-carbohydrazide (16c). White solid, $1.70 \mathrm{~g}(92 \%)$ yield, $\mathrm{mp} 122-123{ }^{\circ} \mathrm{C}, \mathrm{R}_{f} 0.56\left(\mathrm{CHCl}_{3}-\mathrm{MeOH}=6: 1\right)$. IR (KBr): 3336,2962 ,2826, 1669, 1612, 1544, 1507, 1475, 1445, 1279, 1118, 1005, 961, 874, 823, 769, $692 \mathrm{~cm}^{-1}$; ${ }^{1} \mathrm{H} \mathrm{NMR}(300 \mathrm{MHz}$, DMSO-d $d_{6}$ ) $\delta: 10.16$ (br. s, 1H, CONH), 7.52 (br. s, 3H, Ph), 7.33 (br. s, 2H, Ph), 4.57 (br. s, 2H, NH N $^{2}, 3.95$ (s, 2H, $\mathrm{CH}_{2}$ Triaz.) 2.15 (s, $\mathrm{CH}_{2} \mathrm{NCH}_{2}$ ), 1.34 (s, $4 \mathrm{H}, \mathrm{s}, \mathrm{CH}_{2} \mathrm{CH}_{2} \mathrm{NCH}_{2} \mathrm{CH}_{2}$ ); ${ }^{13} \mathrm{C}$ NMR (75.5 MHz, DMSO-d 6 ) $\delta: 159.4,149.2$, $140.5,136.9,131.5,129.4,126.6,120.4,111.7,53.1,45.9,23.0$. HRMS (ESI) $m / z$ for $\mathrm{C}_{16} \mathrm{H}_{19} \mathrm{~N}_{8} \mathrm{O}_{3}(\mathrm{M}+\mathrm{H})^{+}: \mathrm{calcd}$ for 371.1575 , found 371.1569 .

General procedure for the synthesis of 5-(cycloalkylamino)methyl-1-(5-oxido-3-phenyl-1,2,5-oxadiazol-5ium-4-yl)-1H-1,2,3-triazole-4-carbonyl azide 17a,b. To a solution of the corresponding hydrazide 16 (5 mmol) in AcOH-dioxane $(30 \mathrm{~mL}, 1: 1 \mathrm{v} / \mathrm{v})$ mixture at $2-6{ }^{\circ} \mathrm{C}$ the solution of $\mathrm{NaNO}_{2}(1.04 \mathrm{~g}, 15 \mathrm{mmol})$ in water $(1.5 \mathrm{~mL})$ was added for $15 \mathrm{~min}$. The reaction mixture was stirred for $3 \mathrm{~h}$, then water $(40 \mathrm{~mL})$ was added dropwise, the precipitate formed was filtered off, washed with water and dried in air.

5-Morpholinomethyl-1-(5-oxido-3-phenyl-1,2,5-oxadiazol-5-ium-4-yl)-1H-1,2,3-triazole-4-carbonyl azide (17a). White solid, $1.83 \mathrm{~g}$ (92\%) yield, $\mathrm{mp} 141-142{ }^{\circ} \mathrm{C}, \mathrm{R}_{f} 0.19\left(\mathrm{CHCl}_{3}\right) . \mathrm{IR}(\mathrm{KBr}): 3448,2815,2159,1687,1611$,

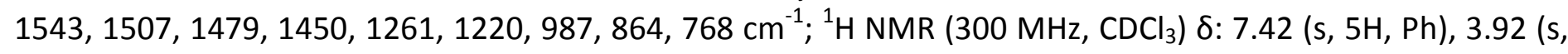




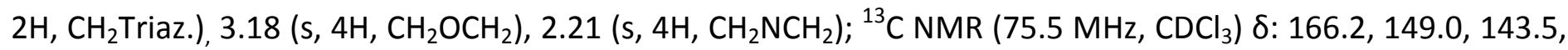
138.3, 131.7, 129.6, 126.5, 120.6, 111.2, 66.2, 53.4, 50.0. HRMS (ESI) $m / z$ for $\mathrm{C}_{16} \mathrm{H}_{16} \mathrm{~N}_{9} \mathrm{O}_{4}(\mathrm{M}+\mathrm{H})^{+}$: calcd for 398.1320, found 398.1309.

5-(4-Ethylpiperazin-1-yl)methyl-1-(5-oxido-3-phenyl-1,2,5-oxadiazol-5-ium-4-yl)-1H-1,2,3-triazole-4-carbonyl azide (17b). White solid, $1.72 \mathrm{~g}(81 \%)$ yield, $\mathrm{mp} 145-165{ }^{\circ} \mathrm{C}$ (dec.), $\mathrm{R}_{f} 0.50\left(\mathrm{CHCl}_{3}-\mathrm{MeOH}=6: 1\right)$. IR ( $\left.\mathrm{KBr}\right): 3483$, $3425,2923,2669,2604,2144,1698,1616,1544,1448,1213,1185,988,771 \mathrm{~cm}^{-1} ;{ }^{1} \mathrm{H} \mathrm{NMR}\left(300 \mathrm{MHz}, \mathrm{CDCl}_{3}\right) \delta$ : 7.65-7.35 (m, 5H, Ph), 4.05 (s, 2H, CH $\mathrm{CH}_{2}$ Triaz.), 3.00-1.70 (m, $\left.10 \mathrm{H}, \mathrm{N}\left(\mathrm{CH}_{2} \mathrm{CH}_{2}\right)_{2} \mathrm{~N}+\mathrm{CH}_{3} \mathrm{CH}_{2} \mathrm{~N}\right), 1,23\left(\mathrm{br} . \mathrm{s}, 3 \mathrm{H}, \mathrm{CH}_{3}\right)$; ${ }^{13} \mathrm{C}$ NMR (75.5 MHz, $\mathrm{CDCl}_{3}$ ) $\delta: 165.9,142.1,138.8,132.0,129.9,126.4,120.6,111.0,52.4,51.1,49.9,9.3$. HRMS (ESI) $m / z$ for $\mathrm{C}_{18} \mathrm{H}_{21} \mathrm{~N}_{10} \mathrm{O}_{3}(\mathrm{M}+\mathrm{H})^{+}$: calcd for 425.1793 , found 425.1788 .

General procedure for the synthesis of 5-(cycloalkylamino)methyl)-1-(5-oxido-3-phenyl-1,2,5-oxadiazol-5ium-4-yl)-1H-1,2,3-triazol-4-carboxamides $\mathbf{5 i , j , l}$. To the solution of azidocarbonyl derivatives 17 a or $17 \mathrm{~b}$ (1 $\mathrm{mmol}$ ) in dioxane $(5 \mathrm{~mL})$ corresponding cycloalkylamine $(2 \mathrm{mmol})$ was added. The reaction mixture was stirred at $20{ }^{\circ} \mathrm{C}$ for 3-10 h until disappearance of initial compound 17 (TLC monitoring). Then water (40 mL) was added, the product was extracted with EtOAc $(2 \times 25 \mathrm{~mL})$, dried over $\mathrm{MgSO}_{4}$ and solvent was evaporated under reduced pressure. Then $\mathrm{Et}_{2} \mathrm{O}(10 \mathrm{~mL})$ was added, the residue was pounded at cooling, the solid formed was filtered off, washed with a small amount of cold $\mathrm{Et}_{2} \mathrm{O}$ and dried in air.

(4-Ethylpiperazin-1-yl)[5-morpholinomethyl-1-(5-oxido-3-phenyl-1,2,5-oxadiazol-5-ium-4-yl)-1H-1,2,3-triazol-4-yl]methanone (5i). White solid, $0.33 \mathrm{~g}(75 \%)$ yield, $\mathrm{mp} 136-137{ }^{\circ} \mathrm{C}, \mathrm{R}_{f} 0.46\left(\mathrm{CHCl}_{3}-\mathrm{MeOH}=6: 1\right) . \mathrm{IR}(\mathrm{KBr})$ :

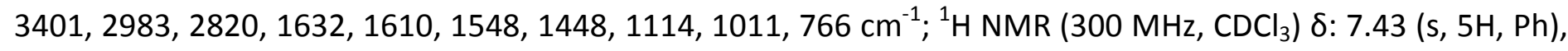
4.10 (s, 2H, CH 2 -Triaz.), 3.81 (s, 4H, $\mathrm{CH}_{2} \mathrm{NCH}_{2}$ Piperazine), 3.18 (s, 4H, $\left.\mathrm{CH}_{2} \mathrm{OCH}_{2}\right), 2.59\left(\mathrm{~s}, 4 \mathrm{H}, \mathrm{CH}_{2} \mathrm{NCH}_{2}\right.$ Piperazine), 2.49 (q, $2 \mathrm{H}, \mathrm{CH}_{2} \mathrm{CH}_{3},{ }^{3}$ J 7.1), 2.24 (s, $\left.4 \mathrm{H}, \mathrm{CH}_{2} \mathrm{NCH}_{2}\right), 1.23$ (t, 3H, $\mathrm{CH}_{2} \mathrm{CH}_{3},{ }^{3}$ J 7.1$) ;{ }^{13} \mathrm{C} \mathrm{NMR}(75.5 \mathrm{MHz}$, $\mathrm{CDCl}_{3}$ ) $\delta: 159.4,149.4,141.7,141.2,131.6,129.5,126.5,120.8,111.4,66.3,53.4,53.3,52.5,52.3,50.3,47.3$, 42.6, 11.9; HRMS (ESI) $m / z$ for $\mathrm{C}_{22} \mathrm{H}_{29} \mathrm{~N}_{8} \mathrm{O}_{4}(\mathrm{M}+\mathrm{H})^{+}$: calcd 469.2306, found 469.2303.

[5-Morpholinomethyl-1-(5-oxido-3-phenyl-1,2,5-oxadiazol-5-ium-4-yl)-1H-1,2,3-triazol-4-yl](pyrrolidinyl)methanone (5j). White solid, $0.35 \mathrm{~g}(82 \%)$ yield, mp126-127 ${ }^{\circ} \mathrm{C}, \mathrm{R}_{f} 0.35\left(\mathrm{CHCl}_{3}\right.$-EtOAc = 4:1). IR (KBr): 3436, 2973, 2826, 1714, 1627, 1610, 1537, 1445, 1115, 1015, 866, 771, $698 \mathrm{~cm}^{-1} ;{ }^{1} \mathrm{H}$ NMR $\left(300 \mathrm{MHz}, \mathrm{CDCl}_{3}\right) \delta: 7.42$ (br. s, 5H, Ph), 4.09 (t, 2H CHNCH in pyrr), 3.92 (s, 2H CH 2 -Triaz.), 3.67 (t, 2H CHNCH in pyrr.), ${ }^{3} J 6.3$ ), 3.18 (br. s, $\left.4 \mathrm{H}, \mathrm{CH}_{2} \mathrm{OCH}_{2}\right), 2.23\left(\mathrm{t}, 4 \mathrm{H}, \mathrm{CH}_{2} \mathrm{NCH}_{2},{ }^{3} \mathrm{~J} 4.1\right), 1.91\left(\mathrm{~m}, 4 \mathrm{H},\left(\mathrm{CH}_{2}\right)_{2}\right) ;{ }^{13} \mathrm{C} \mathrm{NMR}\left(75.5 \mathrm{MHz}, \mathrm{CDCl}_{3}\right) \delta: 159.1,149.4$, 141.5, 131.4, 129.3, 126.4, 120.9, 111.4, 66.2, 53.3, 50.3, 49.0, 46.9, 26.5, 23.8; HRMS (ESI) $m / z$ for $\mathrm{C}_{20} \mathrm{H}_{24} \mathrm{~N}_{7} \mathrm{O}_{4}$ $(\mathrm{M}+\mathrm{H})^{+}$: calcd 426.1884, found 426.1878 .

[5-(4-Ethylpiperazin-1-yl)methyl-1-(5-oxido-3-phenyl-1,2,5-oxadiazol-5-ium-4-yl)-1H-1,2,3-triazol-4-yl](morpholino)methanone (5I). White solid, $0.24 \mathrm{~g}$ (51\%) yield, mp134-135 ${ }^{\circ} \mathrm{C}, \mathrm{R}_{f} 0.42\left(\mathrm{CHCl}_{3}-\mathrm{MeOH}=10: 1\right)$. IR (KBr): 3449, 2817, 1621, 1606, 1543, 1447, 1229, 1116, 1010, 764, $690 \mathrm{~cm}^{-1} ;{ }^{1} \mathrm{H}$ NMR $\left(300 \mathrm{MHz}, \mathrm{CDCl}_{3}\right) \delta: 7.42$ (s, 5H, Ph), 4.14 (s, 2H, CH -Triaz.), 3.80 (br s, 8H, Morpholine), 2.27-2.21 (m, 8H, Piperazine), 2.16 (q, $2 \mathrm{H}$, $\mathrm{CH}_{2} \mathrm{CH}_{3}, 3$ J 7.0), 0.95 (t, 3H, $\left.\mathrm{CH}_{2} \mathrm{CH}_{3},{ }^{3} \mathrm{~J} 7.0\right) ;{ }^{13} \mathrm{C} \mathrm{NMR}\left(75.5 \mathrm{MHz}, \mathrm{CDCl}_{3}\right.$ ) $\delta: 159.4,149.3,142.4,131.3,129.2$, $126.3,120.9,111.5,67.2,66.9,53.0,52.1,50.3,47.9,43.0,11.8$; HRMS (ESI) $m / z$ for $\mathrm{C}_{22} \mathrm{H}_{29} \mathrm{~N}_{8} \mathrm{O}_{4}(\mathrm{M}+\mathrm{H})^{+}: \mathrm{calcd}$ 469.2306, found 469.2302 .

Synthesis of 5-morpholinomethyl-1-(5-oxido-3-phenyl-1,2,5-oxadiazol-5-ium-4-yl)-1H-1,2,3-triazole-4-carboxylic acid $(5 \mathrm{~g})$. Compound $17 \mathrm{a}(1.99 \mathrm{~g}, 5 \mathrm{mmol})$ was added to the solution of $\mathrm{NaOH}(0.50 \mathrm{~g}, 12.5 \mathrm{mmol})$ in the mixture of water $(40 \mathrm{~mL})$ and dioxane $(12 \mathrm{~mL})$. The resulted suspension was stirred until disappearance of the compound 17a for $5 \mathrm{~h}$. After filtration reaction mixture was acidified to $\mathrm{pH} 7$ by addition of AcOH. Then water $(100 \mathrm{~mL})$ was added, the precipitate formed was filtered off, washed with water and dried in air. White solid, $1.72 \mathrm{~g}(92 \%)$ yield, $\mathrm{mp} 168-170{ }^{\circ} \mathrm{C}, \mathrm{R}_{f} 0.65\left(\mathrm{CHCl}_{3}-\mathrm{MeOH}=6: 1\right) . \mathrm{IR}(\mathrm{KBr}): 3391,3198,2851,1714,1660,1613$, $1541,1507,1476,1448,1293,1116,1007,865,771 \mathrm{~cm}^{-1} ;{ }^{1} \mathrm{H}$ NMR $\left(300 \mathrm{MHz}, \mathrm{DMSO}-d_{6}\right) \delta: 11.17$ (br s, $\left.1 \mathrm{H}, \mathrm{OH}\right)$, 
7.59 (br s, 3H, Ph), 7.43 (br s, $2 \mathrm{H}, \mathrm{Ph}$ ), 3.92 (s, 2H, CH NMR $\left.(75.5 \mathrm{MHz} \text {, DMSO-d })_{6}\right) \delta: 158.3,149.1,140.5,138,7,131,6,129,5,126,6,120,3,111.6,65,6,55.6,52.8$. HRMS (ESI) $m / z$ for $\mathrm{C}_{16} \mathrm{H}_{17} \mathrm{~N}_{6} \mathrm{O}_{5}(\mathrm{M}+\mathrm{H})^{+}$: calcd 373.1259 , found 373.1252 .

Synthesis of $\mathrm{N}^{\prime}$-(5-Bromo-1-ethyl-3-oxoindolin-2-ylidene)-5-morpholinomethyl-1-(5-oxido-3-phenyl-1,2,5oxadiazol-5-ium-4-yl)-1H-1,2,3-triazole-4-carbohydrazide (5h). A solution of hydrazide $16 \mathrm{a}(0.39 \mathrm{~g}, 1 \mathrm{mmol})$ and 5-bromo-1-ethylisatin $(0.32 \mathrm{~g}, 1 \mathrm{mmol})$ in a mixture of EtOH $(15 \mathrm{~mL})$ and $\mathrm{AcOH}(0.5 \mathrm{~mL})$ was refluxed for $3.5 \mathrm{~h}$. After cooling to room temperature the precipitate formed was filtered off, washed with $\mathrm{EtOH}(10 \mathrm{~mL})$ and dried in air. Yellow solid, $0.54 \mathrm{~g}(77 \%)$ yield, mp151-152 ${ }^{\circ} \mathrm{C}, \mathrm{R}_{f} 0.17\left(\mathrm{CHCl}_{3}\right) . \mathrm{IR}(\mathrm{KBr}): 3574,3475,2850$, $1707,1692,1613,1507,1475,1342,1182,1110,934,666,511,444 \mathrm{~cm}^{-1} ;{ }^{1} \mathrm{H}$ NMR $\left(300 \mathrm{MHz}, \mathrm{CDCl}_{3}\right) \delta: 14.54$ (s, 1H, NH), 7.98 (s, 1H, Het), 7.53 (d, 1H, Het, ${ }^{3} \mathrm{~J} 8.1$ ), 7.46 (s, 5H, Ph), 6.82 (d, 1H, Het, $\left.{ }^{3} \mathrm{~J} 8.1\right), 4.07$ (s, 2H, $\mathrm{CH}_{2}$ Triaz.), 3.85 (q, 2H, NCH $\mathrm{CH}_{3}, 3$ J 7.0), 3.21 (s, 4H, $\mathrm{CH}_{2} \mathrm{OCH}_{2}$ ), 2.27 (s, $4 \mathrm{H}, \mathrm{CH}_{2} \mathrm{NCH}_{2}$ ), 1.34 (t, 3H, $\mathrm{CH}_{3}, 3 \mathrm{~J} 7.0$ );

${ }^{13} \mathrm{C} \mathrm{NMR}\left(75.5 \mathrm{MHz}, \mathrm{CDCl}_{3}\right)$ $\delta: 160.7,157.0,142.6,141.8,138.5,134.4,131.6,129.5,126.5,125.2,120.6,116.3$, 110.7, 66.3, 53.3, 49.7, 34.8, 12.8; HRMS (ESI) $m / z$ for $\mathrm{C}_{26} \mathrm{H}_{25}{ }^{79} \mathrm{BrN}_{9} \mathrm{O}_{5}(\mathrm{M}+\mathrm{H})^{+}$: calcd 622.1157, found 622.1147. Synthesis of 1-(5-oxido-3-phenyl-1,2,5-oxadiazol-5-ium-4-yl)-5-(pyrrolidin-1-yl)methyl-1H-1,2,3-triazole-4carboxylic acid $(5 \mathrm{~m})$. To a solution of the hydrazide $16 \mathrm{c}(1.48 \mathrm{~g}, 4 \mathrm{mmol})$ in a mixture of dioxane $(4 \mathrm{~mL})$ and AcOH $(4 \mathrm{~mL})$ at $2-7{ }^{\circ} \mathrm{C}$ a solution of $\mathrm{NaNO}_{2}(420 \mathrm{mg}, 6 \mathrm{mmol})$ in water $(1.6 \mathrm{~mL})$ was added over $12 \mathrm{~min}$. The reaction mixture was stirred at the same temperature for $1 \mathrm{~h}$, the temperature was allowed to warm to $20{ }^{\circ} \mathrm{C}$ and the stirring was continued for $10 \mathrm{~h}$. Then another portion of $\mathrm{NaNO}_{2}(420 \mathrm{mg}, 6 \mathrm{mmol})$ was added and the reaction mixture was stirred for $10 \mathrm{~h}$ until disappearance of the initial hydrazide 16c (TLC monitoring). The precipitate formed was filtered off, washed with water and dried in air. White solid, $1.16 \mathrm{~g}(81 \%)$ yield, $\mathrm{mp} 134-135{ }^{\circ} \mathrm{C}, \mathrm{R}_{f} 0.52$ (MeOH). IR (KBr): 3429, 1644, 1615, 1550, 1480, 1449, 1388, 1279, 1063, 827, 774, 690, $514 \mathrm{~cm}^{-1} ;{ }^{1} \mathrm{H}$ NMR (300 MHz, DMSO-d $\left.d_{6}\right) \delta: 7.52$ (s, 3H, Ph), 7.37 (2H, s, Ph), 4.44 (s, 2H, CH${ }_{2}$ Triaz.), 3.50 (br. s, $1 \mathrm{H}, \mathrm{OH}), 2.75\left(\mathrm{~s}, 4 \mathrm{H}, \mathrm{CH}_{2} \mathrm{NCH}_{2}\right), 1.69\left(\mathrm{~s}, 4 \mathrm{H},\left(\mathrm{CH}_{2} \mathrm{CH}_{2} \mathrm{NCH}_{2} \mathrm{CH}_{2}\right) ;{ }^{13} \mathrm{C} \mathrm{NMR}\left(75.5 \mathrm{MHz}, \mathrm{DMSO}-d_{6}\right) \delta: 161.2,148.7\right.$, $141.5,137.3,131.4,129.2,127.3,120.4,111.8,52.5,46.2,23.0$. HRMS (ESI) $m / z$ for $\mathrm{C}_{16} \mathrm{H}_{17} \mathrm{~N}_{6} \mathrm{O}_{5}(\mathrm{M}+\mathrm{H})^{+}$: calcd 357.1306, found 357.1300.

Synthesis of 5-phenyl-1-(5-oxido-3-phenyl-1,2,5-oxadiazol-5-ium-4-yl)-1H-1,2,3-triazole-4-carboxylic acid (18). A solution of $\mathrm{NaHCO}_{3}(640 \mathrm{mg}, 7.6 \mathrm{mmol})$ in water $(30 \mathrm{~mL})$ was added to the ester $12 \mathrm{a}(720 \mathrm{mg}, 1.9$ $\mathrm{mmol})$. The resulting mixture was refluxed for $2 \mathrm{~h}$, then cooled to room temperature, treated with dilute hydrochloric acid, extracted with EtOAc $(3 \times 20 \mathrm{~mL})$ and dried over $\mathrm{MgSO}_{4}$. Light yellow solid. Yield $0.60 \mathrm{~g}(41 \%)$, $\mathrm{mp87}-88^{\circ} \mathrm{C}, \mathrm{R}_{f} 0.53\left(\mathrm{CHCl}_{3}\right)$. IR ( $\left.\mathrm{KBr}\right): 3068,2986,1740,1604,1538,1498,1471,1445,1422,1303,1266,1200$, $1060,1005,959,846,818,762 \mathrm{~cm}^{-1} .{ }^{1} \mathrm{H}$ NMR (300 MHz, DMSO- $\left.d_{6}\right)$ 8: 8.06-8.04 (m, 3H, Ph), 7.68-7.61 (m, 2H, $\mathrm{Ph})$, 7.55-7.48 (m, 5H, Ph), 2.48 (br. s, OH); ${ }^{13} \mathrm{C}$ NMR (75.5 MHz, DMSO-d $\left.d_{6}\right) \delta: 159.3,148.5,142.7,136.5,131.4$, $131.3,130.6,130.0,129.3,128.2,127.1,122.9,119.8,119.7,111.2 ; \mathrm{HRMS}(\mathrm{ESI}) \mathrm{m} / z$ for $\mathrm{C}_{17} \mathrm{H}_{12} \mathrm{~N}_{5} \mathrm{O}_{4}(\mathrm{M}+\mathrm{H})^{+}$: calcd 350.0884 , found 350.0877

\section{General procedure for synthesis of 3-phenyl-4-(5-R-1H-1,2,3-triazol-1-yl)-1,2,5-oxadiazole 2-oxides 5k,n}

The corresponding carboxylic acid $5 \mathrm{~g}$ or $\mathbf{1 8}(2 \mathrm{mmol})$ was dissolved in acetic acid $(20 \mathrm{~mL})$, the resulting solution was refluxed for $30 \mathrm{~min}$ for compound $\mathbf{5 g}$ or for $3 \mathrm{~h}$ for compound $\mathbf{1 8}$. Then $\mathrm{AcOH}$ was evaporated under reduced pressure. The residue was purified using crystallization from EtOH for compound $\mathbf{5 k}$ or flash chromatography (eluent $\mathrm{CHCl}_{3}$-EtOAc $=4: 1$ ) for compound $5 \mathbf{n}$.

4-[5-(Morpholinomethyl)-1H-1,2,3-triazol-1-yl]-3-phenyl-1,2,5-oxadiazole 2-oxide(5k). Light grey solid, $0.51 \mathrm{~g}$ (77\%) yield, mp126-127 ${ }^{\circ} \mathrm{C}, \mathrm{R}_{f} 0.41\left(\mathrm{CHCl}_{3}\right.$-EtOAc = 4:1). IR (KBr): 3434, 2980, 2860, 2798, 1621, 1551, 1511, 1446, 1286, 1243, 1117, 1074, 867, 839, $769 \mathrm{~cm}^{-1} ;{ }^{1} \mathrm{H}$ NMR (300 MHz, CDCl$)$ ): $7.76(\mathrm{~s}, 1 \mathrm{H}, \mathrm{CH}), 7.44-7.41(\mathrm{~m}$, $5 \mathrm{H}, \mathrm{Ph}$ ), 3.55 (s, 2H, CH $\mathrm{CH}_{2}$ Triaz.), $3.23\left(\mathrm{~s}, 4 \mathrm{H}, \mathrm{CH}_{2} \mathrm{OCH}_{2}\right), 2.20\left(\mathrm{~s}, 4 \mathrm{H}, \mathrm{CH}_{2} \mathrm{NCH}_{2}\right) ;{ }^{13} \mathrm{C} \mathrm{NMR}\left(75.5 \mathrm{MHz}^{\mathrm{CDCl}}\right)_{3} \delta$ : 
149.2 , 137.4, 133.9, 131.2, 129.3, 126.7, 120.9, 111.2, 66.4, 53.2, 50.4; HRMS (ESI) $m / z$ for $\mathrm{C}_{15} \mathrm{H}_{17} \mathrm{~N}_{6} \mathrm{O}_{3}(\mathrm{M}+\mathrm{H})^{+}$: calcd 329.1357, found 329.1359.

3-Phenyl-4-(5-phenyl-1H-1,2,3-triazol-1-yl)-1,2,5-oxadiazole 2-oxide (5n). Light orange solid, $0.29 \mathrm{~g}$ (55\%) yield, $\mathrm{mp91}-92{ }^{\circ} \mathrm{C}, \mathrm{R}_{f} 0.15\left(\mathrm{CHCl}_{3}\right)$. IR ( $\left.\mathrm{KBr}\right):$ 3434, 3072, 2677, 2563, 1688, 1604, 1454, 1424, 1327, 1292, 1180, $1128,1073,1027,935,810,762,708 \mathrm{~cm}^{-1} .{ }^{1} \mathrm{H}$ NMR $\left(300 \mathrm{MHz}\right.$, acetone-d $\left.d_{6}\right) \delta: 7.96-7.92(\mathrm{~m}, 3 \mathrm{H}, \mathrm{Ph}) .7 .65-7.58$ $(\mathrm{m}, 2 \mathrm{H}, \mathrm{Ph})$ 7.54-7.46 (m, 5H, Ph), $7.33(\mathrm{~s}, 1 \mathrm{H}, \mathrm{CH}) ;{ }^{13} \mathrm{C}$ NMR $\left(50.3 \mathrm{MHz}\right.$, acetone- $\left.d_{6}\right) \delta:$ 167.3, 133.4, 132.9, $131.3,130.8,130.2,129.3,129.1,128.5,128.2,127.1,126.7$; HRMS (ESI) $\mathrm{m} / z$ for $\mathrm{C}_{16} \mathrm{H}_{12} \mathrm{~N}_{5} \mathrm{O}_{2}(\mathrm{M}+\mathrm{H})^{+}: \mathrm{calcd}$ 306.0986, found 306.0980 .

\section{Cytotoxicity in vitro}

The $I C_{50}$ values of the synthesized compounds against cells were determined by the MTT method. ${ }^{42}$ A549, HCT116, HeLa, MCF7, RD and HEK293 cells were seeded at $1.0 \times 10^{4}$ cells $/ 200 \mu \mathrm{L}$ in 96-well plates and incubated at $37{ }^{\circ} \mathrm{C}$ in a humidified atmosphere with $5 \% \mathrm{CO}_{2}$. After $24 \mathrm{~h}$ of preincubation, the various concentrations of the tested compounds (100-1.56 $\mu \mathrm{M})$ were added into each well, and these cells were incubated under similar conditions for $72 \mathrm{~h}$. All compounds were dissolved in DMSO. The final DMSO concentration in each well did not exceed $1 \%$ and was not toxic for the cells. The wells with a specific cell culture containing $1 \%$ DMSO solution in the medium were monitored as control. After incubation, 20 mM MTT reagent (3-(4,5-dimethylthiazol-2-yl)-2,5-diphenyltetrazolium bromide), at a final concentration of $5 \mathrm{mg} / \mathrm{mL}$, was added into each well, and the cells were incubated for another $2 \mathrm{~h}$. The medium was removed and $100 \mu \mathrm{L}$ DMSO was added to each well. The optical density was measured at $544 \mathrm{~nm}$ minus background absorption at $620 \mathrm{~nm}$ using the Victor3 (Perkin Elmer) microplate reader. Concentrations $\left(\mathrm{IC}_{50}\right)$ were calculated according to the dose-dependent inhibition curves with GraphPad Prism 7 software. The experiments were carried out in triplicate.

NO release assay. The test molecule $(0.1 \mathrm{mmol})$ was dissolved in DMSO $(50 \mathrm{~mL}) .20 \mu \mathrm{L}$ aliquot of the resulted solution was diluted with phosphate buffer solution $(180 \mu \mathrm{L}, \mathrm{pH} 7.4$, containing $2 \mu \mathrm{mol} \mathrm{L}$-cysteine). The final concentration of the furoxan derivative was $2 \cdot 10^{-4} \mathrm{M}$. The mixture was incubated at $37^{\circ} \mathrm{C}$ for $1 \mathrm{~h} .50 \mu \mathrm{L}$ aliquot of the Griess reagent (prepared by mixing sulfanilamide $(4 \mathrm{~g}), N$-naphthylethylenediamine dihydrochloride $(0.2$ g) and $85 \% \mathrm{H}_{3} \mathrm{PO}_{4}(10 \mathrm{~mL}$ ) in distilled and deionized water (final volume $100 \mathrm{~mL}$ )) was added and incubated for $10 \mathrm{~min}$ at $37{ }^{\circ} \mathrm{C}$. UV absorbance at $540 \mathrm{~nm}$ was measured using a Multiskan GO Microplate Photometer and calibrated using a standard curve prepared from standard solutions of $\mathrm{NaNO}_{2}$ to give the nitrite concentration. All measurements were made in triplicate. No significant NO release was measured at the absence of Lcysteine.

\section{Acknowledgements}

This work was supported by the Russian Science Foundation (Project No 14-50-00126).

\section{Supplementary Material}

Table S1 containing cytotoxic activity data and copies of NMR spectra of newly synthesized compounds. 


\section{References}

1. Nicolaou, K. C.; Hale, C. R. H.; Nilewski, C.; Ioannidou, H. A. Chem. Soc. Rev. 2012, 41, 5185-5238. https://doi.org/10.1039/c2cs35116a

2. Ananikov, V. P.; Khokhlova, E. A.; Egorov, M. P.; Sakharov, A. M.; Zlotin, S. G.; Kucherov, A. V.; Kustov, L. M.; Gening, M. L.; Nifantiev, N. E. Mendeleev Commun. 2015, 25, 75-82.

https://doi.org/10.1016/j.mencom.2015.03.001

3. Zlotin, S. G.; Churakov, A. M.; Luk'yanov, O. A.; Makhova, N. N.; Sukhorukov, A. Yu.; Tartakovsky, V. A. Mendeleev Commun. 2015, 25, 399-409. https://doi.org/10.1016/i.mencom.2015.11.001

4. Gasco, A.; Fruttero, R.; Sorba, G.; Di Stilo, A.; Calvino, R. Pure Appl. Chem. 2004, 76, 973-981. https://doi.org/10.1351/pac200476050973

5. Fershtat, L. L.; Makhova, N. N. ChemMedChem 2017, 12, 622-638. https://doi.org/10.1002/cmdc.201700113

6. Cheng, R.; Ridnour, L. A.; Glynn, S. A.; Switzer, C. H.; Flores-Santana, W.; Hussain, P.; Thomas, D. D.; Ambs, S.; Harris, C. C.; Wink, D. A. In Nitric Oxide and Cancer. Prognosis, Prevention and Therapy; Bonavida, B. Ed.; Springer: New York, 2010; ch. 1, pp 3-20.

https://doi.org/10.1007/978-1-4419-1432-3 1

7. Granik, V. G.; Grigoriev, N. B. Nitric oxide (NO). New Route to Drug Design [in Russian]; Vuzovskaya Kniga, Moscow, 2004.

8. Gasco, A.; Schoenafinger, K. In Nitric Oxide Donors: For Pharmaceutical and Biological Applications; Wang, P. G.; Cai, T. B.; Taniguchi, N. Eds.; Wiley-VCH: Weinheim, 2005, pp 131-175.

https://doi.org/10.1002/3527603751.ch6

9. Fershtat, L. L.; Makhova, N. N. Russ. Chem. Rev. 2016, 85, 1097-1145. https://doi.org/10.1070/RCR4619

10. Ferioli, R.; Folco, G. C.; Ferretti, C.; Gasco, A. M.; Medana, C.; Fruttero, R.; Civelli, M.; Gasco, A. Br. J. Pharmacol. 1995, 114, 816-820.

https://doi.org/10.1111/j.1476-5381.1995.tb13277.x

11. Kots, A. Ya.; Grafov, M. A.; Khropov, Yu. V.; Betin, V. L.; Belushkina, N. N.; Busygina, O. G.; Yazykova, M. Yu.; Ovchinnikov, I. V.; Kulikov, A. S.; Makhova, N. N.; Medvedeva, N. A.; Bulargina T. V.; Severina, I. S. Br. J. Pharmacol. 2000, 129, 1163-1177.

https://doi.org/10.1038/sj.bjp.0703156

12. Schiefer, I. T.; VandeVrede, L.; Fa, M.; Arancio, O.; Thatcher, G. R. J. J. Med. Chem. 2012, 55, 3076-3087. https://doi.org/10.1021/jm201504s

13. Aguirre, G.; Boiani, M.; Cerecetto, H.; Fernandez, M.; Gonzalez, M.; Leon, E.; Pintos, C.; Raymondo, S.; Arredondo, C.; Pacheco, J. P.; Basombro, M. A. Pharmazie 2006, 61, 54.

14. Zhao, J.; Gou, S.; Sun, Y.; Fang, L.; Wang, Z. Inorg. Chem. 2012, 51, 10317-10324. https://doi.org/10.1021/ic301374z

15. Mott, B. T.; Cheng, K. C.-C.; Guha, R.; Kommer, V. P.; Williams, D. L.; Vermeire, J. J.; Cappello, M.; Maloney, D. J.; Rai, G.; Jadhav, A.; Simeonov, A.; Inglese, J.; Posner, G. H.; Thomas, C. J. Med. Chem. Commun. 2012, 3, 1505-1511.

https://doi.org/10.1039/c2md20238g

16. Dos Santos, J. L.; Lanaro, C.; Chelucci, R. C.; Gambero, S.; Bosquesi, P. L.; Reis, J. S.; Lima, L. M.; Cerecetto, H.; Gonzalez, M.; Costa, F. F.; Chung, M. C. J. Med. Chem. 2012, 55, 7583-7592. 
https://doi.org/10.1021/jm300602n

17. Rai, G.; Thomas, C. J.; Leister, W.; Maloney, D. J. Tetrahedron Lett. 2009, 50, 1710-1713. https://doi.org/10.1016/j.tetlet.2009.01.120

18. Thatcher, G. R. J.; Nicolescu, A. C.; Bennett, B. M.; Toader, V. Free Radical Biol. Med. 2004, 37, 1122-1143. https://doi.org/10.1016/j.freeradbiomed.2004.06.013

19. Lazzarato, L.; Cena, C.; Rolando, B.; Marini, E.; Lolli, M. L.; Guglielmo, S.; Guaita, E.; Morini, G.; Coruzzi, G.; Fruttero, R.; Gasco, A. Bioorg. Med. Chem. 2011, 19, 5852-5860.

https://doi.org/10.1016/i.bmc.2011.08.018

20. Borretto, E.; Lazzarato, L.; Spallotta, F.; Cencioni, C.; D’Alessandra, Yu.; Gaetano, C.; Fruttero, R.; Gasco, A. ACS Med. Chem. Lett. 2013, 4, 994-999.

https://doi.org/10.1021/ml400289e

21. Guglielmo, S.; Cortese, D.; Vottero, F.; Rolando, B.; Kommer, V. P.; Williams, D. L.; Fruttero, R.; Gasco, A. Eur. J. Med. Chem. 2014, 84, 135-145.

https://doi.org/10.1016/j.ejmech.2014.07.007

22. Gu, X.; Huang, Z.; Ren, Z.; Tang, X.; Xue, R.; Luo, X.; Peng, S.; Peng, H.; Lu, B.; Tian, J.; Zhang, Y. J. Med. Chem. 2017, 60, 928-940.

https://doi.org/10.1021/acs.jmedchem.6b01075

23. Nortcliffe, A.; Ekstrom, A. G.; Black, J. R.; Ross, J. A.; Habib, F. K.; Botting, N. P.; O'Hagan, D. Bioorg. Med. Chem. 2014, 22, 756-761.

https://doi.org/10.1016/i.bmc.2013.12.014

24. Zhao, N.; Tian, K.; Cheng, K.; Han, T.; Hu, X.; Li, D.; Li, Z.; Hua, H. Bioorg. Med. Chem. 2016, 24, $2971-2978$. https://doi.org/10.1016/j.bmc.2016.05.001

25. Fang, Y.; Wang, R.; He, M.; Huang, H.; Wang, Q.; Yang, Z.; Li, Y.; Yang, S.; Jin, Y. Bioorg. Med. Chem. Lett. 2017, 27, 98-101.

https://doi.org/10.1016/j.bmcl.2016.11.021

26. Fruttero, R.; Crosetti, M.; Chegaev, K.; Guglielmo, S.; Gasco, A.; Berardi, F.; Niso, M.; Perrone, R.; Panaro, M. A.; Colabufo, N. A. J. Med. Chem. 2010, 53, 5467-5475.

https://doi.org/10.1021/jm100066y

27. Fershtat, L. L.; Epishina, M. A.; Kulikov, A. S.; Struchkova, M. I.; Makhova, N. N. Chem. Heterocycl. Compd. 2015, 51, 176-186.

https://doi.org/10.1007/s10593-015-1678-5

28. Fershtat, L. L.; Ananyev, I. V.; Makhova, N. N. RSC Adv. 2015, 5, 47248-47260. https://doi.org/10.1039/C5RA07295F

29. Fershtat, L. L.; Larin, A. A.; Epishina, M. A.; Ovchinnikov, I. V.; Kulikov, A. S.; Ananyev, I. V.; Makhova, N. N. RSC Adv. 2016, 6, 31526-31539.

https://doi.org/10.1039/C6RA05110C

30. Fershtat, L. L.; Epishina, M. A.; Kulikov, A. S.; Ovchinnikov, I. V.; Ananyev, I. V.; Makhova, N. N. Tetrahedron Lett. 2016, 57, 4268-4272.

https://doi.org/10.1016/i.tetlet.2016.08.011

31. Fujii, S.; Ohta, K.; Goto, T.; Kagechika, H.; Endo, Y. Bioorg. Med. Chem. 2009, 17, 344-350. https://doi.org/10.1016/j.bmc.2008.10.060

32. Khoshneviszadeh, M.; Ghahremani, M. H.; Foroumadi, A.; Miri, R.; Firuzi, O.; Madadkar-Sobhani, A.; Edraki, N.; Parsa, M.; Shafiee, A. Bioorg. Med. Chem. 2013, 21, 6708-6717.

https://doi.org/10.1016/i.bmc.2013.08.009 
33. Grishko, V. V.; Tolmacheva, I. A.; Nebogatikov, V. O.; Galaiko, N. V.; Nazarov, A. V.; Dmitriev, M. V.; Ivshina, I. B. Eur. J. Med. Chem. 2017, 125, 629-639.

https://doi.org/10.1016/i.ejmech.2016.09.065

34. Fershtat, L. L.; Ashirbaev, S. S.; Kulikov, A. S.; Kachala, V. V.; Makhova, N. N. Mendeleev Commun. 2015, 25, 257-259.

https://doi.org/10.1016/i.mencom.2015.07.007

35. Ladani, G. G.; Patel, M. P. New J. Chem. 2015, 39, 9848-9857.

https://doi.org/10.1039/C5NJ02566D

36. Karki, R.; Jun, K.-Y.; Kadayat, T. M.; Shin, S.; Bahadur, T.; Magar, T.; Bist, G.; Shrestha, A.; Na, Y.; Kwon, Y.; Lee, E.-S. Eur. J. Med. Chem. 2016, 113, 228-245.

https://doi.org/10.1016/j.ejmech.2016.02.050

37. Fershtat, L. L.; Struchkova, M. I.; Goloveshkin, A. S.; Bushmarinov, I. S.; Makhova, N. N. Heteroat. Chem. 2014, 25, 226-237.

https://doi.org/10.1002/hc.21166

38. Fershtat, L. L.; Epishina, M. A.; Ovchinnikov, I. V.; Makhova, N. N. Chem. Heterocycl. Compd. 2015, 51, 754-759.

https://doi.org/10.1007/s10593-015-1771-9

39. Fischer, D.; Klapötke, T. M.; Stierstörfer, J. Eur. J. Inorg. Chem. 2014, 5808-5811. https://doi.org/10.1002/ejic.201402960

40. Kulikov, A. S.; Epishina, M. A.; Batog, L. V.; Rozhkov, V. Yu.; Makhova, N. N.; Konyushkin, L. D.; Semenova, M. N.; Semenov, V. V. Russ. Chem. Bull., Int. Ed. 2013, 62, 836-843.

https://doi.org/10.1007/s11172-013-0113-2

41. Fershtat, L. L.; Radzhabov, M. R.; Romanova, A. A.; Ananyev, I. V.; Makhova, N. N. Arkivoc 2017, (iii), 140150.

42. Mosmann, T. J. Immunol. Methods 1983, 65, 55-63.

https://doi.org/10.1016/0022-1759(83)90303-4 